\title{
New Curculionoidea (Coleoptera) records for Canada
}

\author{
Hume Douglas', Patrice Bouchard ${ }^{2}$, Robert S. Anderson ${ }^{3}$, Pierre de Tonnancour ${ }^{4}$, \\ Robert Vigneault ${ }^{5}$, Reginald P. Webster ${ }^{6}$
}

I Entomology, Ottawa Plant Laboratories, Canadian Food Inspection Agency, Building 18, 960 Carling Avenue, Ottawa, ON, Canada, K1A OC6 2 Canadian National Collection of Insects, Arachnids and Nematodes, Agriculture and Agri-Food Canada, 960 Carling Avenue, Ottawa, Ontario, Canada, K1A OC6 3 Canadian Museum of Nature, P.O. Box 3443, Station D, Ottawa, Ontario, Canada, K1P 6P4 4 22, 5e avenue, TerrasseVaudreuil, Quebec, Canada, J7V $3 P 5516$ Mont St-Pierre, Oka, Quebec, Canada, JoN 1 EO 624 Mill Stream Drive, Charters Settlement, New Brunswick, Canada, E3C $1 X 1$

Corresponding author: Patrice Bouchard (Patrice.Bouchard@agr.gc.ca)

Academic editor: Christopher Majka | Received 11 January 2013 | Accepted 15 May 2013 | Published 13 June 2013

Citation: Douglas H, Bouchard P, Anderson RS, de Tonnancour P, Vigneault R, Webster RP (2013) New Curculionoidea (Coleoptera) records for Canada. ZooKeys 309: 13-48. doi: 10.3897/zookeys.309.4667

\begin{abstract}
The following species of Curculionoidea are recorded from Canada for the first time, in ten cases also representing new records at the generic level: Ischnopterapion (Ischnopterapion) loti (Kirby, 1808); Stenopterapion meliloti (Kirby, 1808) (both Brentidae); Atrichonotus taeniatulus (Berg, 1881); Barinus cribricollis (LeConte, 1876); Caulophilus dubius (Horn, 1873); Cionus scrophulariae (Linnaeus, 1758); Cryptorhynchus tristis LeConte, 1876; Cylindrocopturus furnissi Buchanan, 1940; Cylindrocopturus quercus (Say, 1832); Desmoglyptus crenatus (LeConte, 1876); Pnigodes setosus LeConte, 1876; Pseudopentarthrum parvicollis (Casey, 1892); Sibariops confinis (LeConte, 1876); Sibariops confusus (Boheman, 1836); Smicronyx griseus LeConte, 1876; Smicronyx lineolatus Casey, 1892; Euwallacea validus (Eichhoff, 1875); Hylocurus rudis (LeConte, 1876); Lymantor alaskanus Wood, 1978; Phloeotribus scabricollis (Hopkins, 1916); Scolytus oregoni Blackman, 1934; Xyleborus celsus Eichhoff, 1868; Xyleborus ferrugineus (Fabricius, 1801); Xylosandrus crassiusculus (Motschulsky, 1866) (all Curculionidae). In addition the following species were recorded for the first time from these provinces and territories: Yukon - Dendroctonus simplex LeConte, 1868; Phloetribus piceae Swaine, 1911 (both Curculionidae); Northwest Territories - Loborhynchapion cyanitinctum (Fall, 1927) (Brentidae); Nunavut - Dendroctonus simplex LeConte, 1868 (Curculionidae); Alberta - Anthonomus tectus LeConte, 1876; Promecotarsus densus Casey, 1892; Dendroctonus ponderosae Hopkins, 1902; Hylastes macer LeConte, 1868; Rhyncolus knowltoni (Thatcher, 1940); Scolytus schevyrewi Semenov Tjan-Shansky, 1902 (all Curculionidae); Saskatchewan - Phloeotribus liminaris (Harris, 1852); Rhyncolus knowltoni (Thatcher, 1940); Scolytus schevyrewi Semenov Tjan-Shansky, 1902 (all Curculionidae); Manitoba - Cosmobaris scolopacea Germar, 1819; Listronotus maculicollis (Kirby, 1837); Listronotus punctiger LeConte, 1876;
\end{abstract}

Copyright Hume Douglas et al. This is an open access article distributed under the terms of the Creative Commons Attribution License 3.0 (CC-BY), which permits unrestricted use, distribution, and reproduction in any medium, provided the original author and source are credited. 
Scolytus schevyrewi Semenov Tjan-Shansky, 1902; Tyloderma foveolatum (Say, 1832); (all Curculionidae); Ontario - Trichapion nigrum (Herbst, 1797); Nanophyes marmoratus marmoratus (Goeze, 1777) (both Brentidae); Asperosoma echinatum (Fall, 1917); Micracis suturalis LeConte, 1868; Orchestes alni (Linnaeus, 1758); Phloeosinus pini Swaine, 1915; Scolytus schevyrewi Semenov Tjan-Shansky, 1902; Xyleborinus attenuatus (Blandford, 1894) (all Curculionidae); Quebec - Trigonorhinus alternatus (Say, 1826); Trigonorhinus tomentosus tomentosus (Say, 1826) (both Anthribidae); Trichapion nigrum (Herbst, 1797); Trichapion porcatum (Boheman, 1839); Nanophyes marmoratus marmoratus (Goeze, 1777) (all Brentidae); Lissorhoptrus oryzophilus Kuschel, 1952 (Brachyceridae); Acalles carinatus LeConte, 1876; Ampeloglypter ampelopsis (Riley, 1869); Anthonomus rufipes LeConte, 1876; Anthonomus suturalis LeConte, 1824; Ceutorhynchus hamiltoni Dietz, 1896; Curculio pardalis (Chittenden, 1908); Cyrtepistomus castaneus (Roelofs, 1873); Larinus planus (Fabricius, 1792); Mecinus janthinus (Germar, 1821); Microhyus setiger LeConte, 1876; Microplontus campestris (Gyllenhal, 1837); Orchestes alni (Linnaeus, 1758); Otiorhynchus ligustici (Linnaeus, 1758); Rhinusa neta (Germar, 1821); Trichobaris trinotata (Say, 1832); Tychius liljebladi Blatchley, 1916; Xyleborinus attenuatus (Blandford, 1894); Xyleborus affinis Eichhoff, 1868 (all Curculionidae); Sphenophorus incongruus Chittenden, 1905 (Dryophthoridae); New Brunswick - Euparius paganus Gyllenhal, 1833; Allandrus populi Pierce, 1930; Gonotropis dorsalis (Thunberg, 1796); Euxenus punctatus LeConte, 1876 (all Anthribidae); Loborhynchapion cyanitinctum (Fall, 1927) (Brentidae); Pseudanthonomus seriesetosus Dietz, 1891; Curculio sulcatulus (Casey, 1897); Lignyodes bischoffi (Blatchley, 1916); Lignyodes horridulus (Casey, 1892); Dietzella zimmermanni (Gyllenhal, 1837); Parenthis vestitus Dietz, 1896; Pelenomus squamosus LeConte, 1876; Psomus armatus Dietz, 1891; Rhyncolus macrops Buchanan, 1946; Magdalis inconspicua Horn, 1873; Magdalis salicis Horn, 1873 (all Curculionidae); Nova Scotia - Dryocoetes autographus (Ratzeburg, 1837); Ips perroti Swaine, 1915; Xyleborinus attenuatus (Blandford, 1894) (all Curculionidae); Prince Edward Island - Dryocoetes caryi Hopkins, 1915 (Curculionidae); Newfoundland - Scolytus piceae (Swaine, 1910) (Curculionidae).

Published records of Dendroctonus simplex LeConte, 1868 from Northwest Territories should be reassigned to Nunavut, leaving no documented record for NWT. Collection data are provided for eight provincial and national records published without further information previously.

\section{Keywords}

Anthribidae, Brachyceridae, Brentidae, Curculionidae, Dyophthoridae, weevils, bark beetles, pests

\section{Introduction}

Routine weevil and bark beetle identifications from plant health surveys, amateur collectors, public inquiries, and museum survey specimens regularly produce new faunal records for Canada, its provinces, and territories. The most recent checklist of the Canadian fauna is McNamara (1991).

The present article presents new findings with associated collection data so that the records may be documented with verifiable voucher specimens. These records are also reflected in the updated checklist of Canadian beetles (Bousquet et al. 2013). The following list of 97 new records are organised according to the family-group classification of Bouchard et al. (2011). We record two Brentidae, and 22 Curculionidae species new to Canada, and 72 new provincial and territorial records; many of these are of beneficial or economic pest species. 


\section{Materials and methods}

Specimens were identified (or identifications confirmed) by recognized specialists in those taxa. These are as follows: Curculionidae (Scolytinae) (Hume Douglas, Donald E. Bright); Curculionidae (other than Scolytinae), Anthribidae, Brachyceridae (Robert S. Anderson, Patrice Bouchard); Brentidae (Apioninae) and Curculionidae (Baridinae) (Jens Prena). Specimens are deposited in collections listed with the specimen data for each species.

All collections listed below were reviewed by one or more authors for undocumented curculionoid records except for DEBU, GLFC, and City of Saskatoon. For these three collections we included only the specimens identified as new by their staff. It is possible that additional undocumented curculionoid records remain in most of the collections listed below.

The use of term adventive used here follows that of Wheeler and Hoebeke (2009). Such adventive species are non-natives with established North American populations, intentionally or accidentally introduced by humans, effectively since the first arrival of Europeans.

\section{Canadian collections that provided material cited here:}

AFC Atlantic Forestry Centre, Natural Resources Canada, Canadian Forest Service, Fredericton, New Brunswick

CCCH Claude Chantal Insect Collection (personal collection), Varennes, Quebec

City of Saskatoon Saskatchewan (Contact Jeff Boone)

CHMS Henri Miquet-Sage Insect Collection (personal collection), Mont-SaintHilaire, Quebec

CMNC Canadian Museum of Nature, Ottawa, Ontario

CNCI Canadian National Collection of Insects, Arachnids, and Nematodes, Agriculture and Agri-Food Canada Research Centre, Ottawa, Ontario

CPTO Pierre de Tonnancour Insect Collection (personal collection), Terrasse-Vaudreuil, Quebec

CRLI René Limoges Insect Collection (personal collection), Montreal, Quebec

CRVI Robert Vigneault Insect Collection (personal collection), Oka, Quebec

CSLA Serge Laplante Insect Collection (personal collection), Gatineau, Quebec

DEBU University of Guelph Insect Collection, Guelph, Ontario

GLFC Great Lakes Forestry Centre, Sault Ste. Marie, Ontario

LEMQ Lyman Entomological Museum, McGill University, Ste-Anne-de-Bellevue, Quebec

NBM New Brunswick Museum, Saint John, New Brunswick

RWC Reginald P. Webster Collection (personal collection), Charters Settlement, New Brunswick 


\section{Results}

1) Family Anthribidae Billberg, 1820

Subfamily Anthribinae Billberg, 1820

Tribe Cratoparini LeConte, 1876

Euparius paganus Gyllenhal, 1833, new to New Brunswick, and new data supporting first records for Canada

Note. This native fungus weevil was recorded "from Quebec to Florida, west to Iowa, Kansas and Texas" by Valentine (1999) without specific details about its distribution within Quebec. We provide those data here for the first time and provide data for specimens from New Brunswick.

Specimen data. New Brunswick: Carleton County, Jackson Falls, Bell Forest, $46.2200^{\circ} \mathrm{N}, 67.7231^{\circ} \mathrm{W}, 17-31 . v i i .2012$, 31.vii-14.viii.2012, C. Alderson \& V. Webster, Lindgren traps in canopy of Juglans cinerea and Tilia americana (1, AFC; 5, RWC). Quebec: MRC Vaudreuil-Soulanges, Rigaud, 12.vii, 14.vii.1998 (2, CRVI); MRC Deux-Montagnes, Oka, Mont St-Pierre, 19.v., 2.viii.2003, UV Light, R. Vigneault (2, CRVI); MRC Les Collines-de-l'Outaouais, Eardley, "petite colline d'Eardley", 17.vi., 25.vii.2003, 14.v.2004, S. Laplante, R. Vigneault, at UV light (3, CSLA; 2 CRVI); MRC Les Collines-de-l'Outaouais, Eardley, "petite colline d'Eardley", 25.vii.2003, S. Laplante, on dead branch of Prunus pensylvanica at night (1, CSLA); MRC Vaudreuil-Soulanges, Terrasse-Vaudreuil, 3.vii.2011 (01:00), white and UV lights, P. de Tonnancour (1, CPTO); MRC Vaudreuil-Soulanges, Terrasse-Vaudreuil, 5.vii.2012 (23:00), white and UV lights, P. de Tonnancour (1, CPTO); MRC Vaudreuil-Soulanges, Terrasse-Vaudreuil, 27.vii.2012, at night (22:30), white and mercury lights, P. de Tonnancour (1, CPTO).

Tribe Stenocerini Kolbe, 1895

Allandrus populi Pierce, 1930, new to New Brunswick

Note. This transcontinental Canadian species appears to be associated with Populus tremuloides (Bright 1993).

Specimen data. New Brunswick: Carleton County, Meduxnekeag Valley Nature Preserve, $46.1907^{\circ} \mathrm{N}, 67.6740^{\circ} \mathrm{W}, 3-17 . v i i .2012$, C. Alderson \& V. Webster, Lindgren trap in Populus tremuloides canopy (3, AFC; 3, RWC); Sunbury County, Gilbert Island, $45.8770^{\circ} \mathrm{N}$, 66.2954 W, 11-29.vi-11.2012, 11-25.vii.2012, 25.vii-8.viii.2012, 8-21.viii.2012 Lindgren traps in Populus tremuloides canopy, C. Alderson, C. Hughes \& V. Webster (4, AFC; 1, NBM; 5, RWC); York County, $15 \mathrm{~km} \mathrm{~W}$ of Tracy, off Rt. $645,45.6848^{\circ} \mathrm{N}, 66.8821^{\circ} \mathrm{W}$, 16-30.vi.2010, Lindgren funnel trap, R. Webster \& C. MacKay (1, RWC). 
Tribe Trigonorhinini Valentine, 1999

Trigonorhinus alternatus (Say, 1826), new to Quebec

Note. This fungus weevil was recorded in Canada from Alberta, Manitoba and Ontario by McNamara (1991).

Specimen data. Quebec: MRC Marguerite-D’Youville, Varennes (Verchères), 6.vi.2003, C. Chantal (1, CCCH).

\section{Trigonorbinus tomentosus tomentosus (Say, 1826), new to Quebec}

Note. This native species was only recorded in Canada from Ontario by McNamara (1991).

Specimen data. Quebec: Montreal, 23.vii.1967, E. J. Kiteley (1, CNCI); Montreal, Sainte- Anne-de-Bellevue, 5.ix.1967, W. Boyle (1, LEMQ; 1, CMNC); Montreal, 21.viii.1968 (1, CNCI); Montreal, 26.viii.1968 (2, CNCI); MRC Vaudreuil-Soulanges, Rigaud (4 mi. S.E.), 4.vii.1972, C. Boyle (1, LEMQ); RCM Le Haut-Saint-Laurent, Cairnside, 29.viii.1981 (1, CNCI); MRC Vaudreuil-Soulanges, Notre-Dame-de-l'Île-Perrot, 27.v.2011 (13:00), 10.vi.2011 (17:00), 2.vii.2011 (15:00), swept from grasses, swept from Scirpus sp., ex. flowers of Lythrum salicaria, P. de Tonnancour (5, CPTO); Montreal, Sainte-Anne-de-Bellevue, 24.viii.2011 (13:00), swept from Ambrosia artemisiifolia, P. de Tonnancour (3, CPTO); Montreal, Sainte-Anne-de-Bellevue, 12.ix.2011 (12:00), swept from Ambrosia artemisiifolia, P. de Tonnancour (1, CPTO); Montreal, Sainte-Anne-de-Bellevue, 5.vii.2012 (13:00), swept from forbs, P. de Tonnancour (1, CPTO).

\section{Tribe Tropiderini Lacordaire, 1865}

\section{Gonotropis dorsalis (Thunberg, 1796), new to New Brunswick}

Note. This transcontinental Canadian species has previously been placed in the genus Tropideres Schönherr.

Specimen data. New Brunswick: York County, Charters Settlement, $45.8395^{\circ} \mathrm{N}, 66.7391^{\circ} \mathrm{W}, 20 . v .2012$, R Webster, on window screen (1, RWC). Fredericton, Odell Park, $45.9571^{\circ} \mathrm{N}, 66.6650^{\circ} \mathrm{W}, 10-26 . v i i .2012$, C. Alderson \& V. Webster, old-growth eastern hemlock forest, Lindgren trap in Tsuga canadensis canopy (2, RWC). 
Tribe Zygaenodini Lacordaire, 1865

Eusphyrus walshii LeConte, 1876, new data supporting first records for Quebec

Note. This species was recorded "from Quebec to Florida, west to Michigan and eastern Texas" by Valentine (1999, not McNamara 1991) without specific details about its distribution within Quebec. We provide data on the occurence of this species in Quebec for the first time.

Specimen data. Quebec: MRC Les Collines-de-l'Outaouais Luskville, Chemin Pilon, 24.vi.2001, C. Chantal (1, CCCH); MRC Marguerite-D’Youville, Varennes (Verchères), 9.ix.2002, C. Chantal (1, CCCH); Longueuil, St-Bruno-de-Montarville, $45.588^{\circ} \mathrm{N}, 73.303^{\circ} \mathrm{W}, 22-29 . v i i .2008$, Projet Défense Nationale, Site 1 Parcelle 4, érablière à caryer, Sante trap, Propylene 100\%, 2008-3-1437 (1, CNCI); MRC Vaudreuil-Soulanges, Rigaud, 1.vii.1993 (15:00), beaten from dead branch of $\mathrm{Ca}$ rya ovata, P. de Tonnancour (1, CPTO); Montreal, Ste-Anne-de-Bellevue, 5.vii.2012 (15:00) on Ulmus americana, P. de Tonnancour (1, CPTO); MRC Marguerite-D'Youville, Contrecoeur, 7.vii.2012, dead branch of Salix sp., P. de Tonnancour (1, CPTO).

\section{Subfamily Choraginae Kirby, 1819}

Tribe Araecerini Lacordaire, 1865

Euxenus punctatus LeConte, 1876, new to New Brunswick

Note. This species was previously known in Canada only from Quebec (McNamara 1991). It is the smallest anthribid in Canada.

Specimen data. New Brunswick: Queens County, Jemseg, $45.8412^{\circ} \mathrm{N}$, 66.1195 $\mathrm{W}, 28 . v i-10 . v i i .2012$, Lindgren trap under Quercus macrocarpa, C. Hughes \& R. Webster (1, RWC).

2) Family Brentidae Billberg, 1820

Subfamily Apioninae Schönherr, 1823

Tribe Apionini Schönherr, 1823

Loborhynchapion cyanitinctum (Fall, 1927), new to New Brunswick, Northwest Territories

Note. This widespread and northern species is recorded from the maritime provinces for the first time. It has been collected on Astragalus (Bright 1993).

Specimen data. New Brunswick: Carleton County, Meduxnekeag Valley Nature Preserve, $46.1891^{\circ} \mathrm{N}, 67.6762^{\circ} \mathrm{W}, 11 . v i .2012$, swept from foliage by river, R.P. Webster (1, RWC). Northwest Territories: Anderson River Delta, Fox Den II, 29.vi-15. vii.1977, D. Shpeley \& G.E. Ball (1, CMNC). 


\section{Ischnopterapion (Ischnopterapion) loti (Kirby, 1808), new to Canada}

Note. This adventive species is broadly distributed in the Palaearctic Region (AlonsoZarazaga 2011). In Canada it feeds on the introduced weed Lotus corniculatus L. (Fabaceae) which is common in eastern Canada and British Columbia (Turkington and Franko 1980). This species may be more widespread in Canada than presently documented because extensive surveys have not been conducted.

Specimen data. Quebec: MRC Vaudreuil-Soulanges, Ville de l'île-Perrot, 17.v.2011, swept from Lotus corniculatus, P. de Tonnancour (2, CNCI; 17, CPTO); MRC Vaudreuil-Soulanges, Ville de l'Île-Perrot, 18.v.2011, swept from Hesperis matronalis, P. de Tonnancour (5, CPTO).

\section{Stenopterapion meliloti (Kirby, 1808), new to Canada}

Note. This adventive species is broadly distributed in the Palaearctic Region (AlonsoZarazaga 2011). In Canada it feeds on the introduced weed Melilotus alba Desr. which is common across Canada (Turkington et al. 1978). This species may be more widespread in Canada than presently documented because extensive surveys have not been conducted.

Specimen data. Quebec: MRC La Vallée-du-Richelieu, Mont-Saint-Hilaire, 7.vi., 23.vi.2004, 18.viii.2007, H. Miquet-Sage (3, CHMS); MRC Vaudreuil-Soulanges, Notre-Dame-de-l'Île-Perrot, 2.iv.2007，12.v.2011 (13:00), 17.v.2011，10.vi.2011, 14.ix.2011, 19.iv.2012 (13:00), under bark of Pinus strobus, swept from Melilotus alba, P. de Tonnancour (2, CNCI; 29, CPTO).

\section{Trichapion nigrum (Herbst, 1797), new to Ontario and Quebec}

Note. This native species was newly recorded in Canada in New Brunswick by Majka et al. (2007a) and feeds on the seeds of Robinia pseudoacacia L. (Fabaceae). That the first specimen was collected over 50 years ago, that the host is widespread, and that this tribe has received little taxonomic attention, all suggest that this species may be more widespread than known.

Specimen data. Ontario: Simcoe Co., Cookstown, Lake Simcoe, 11.vi.1962, S621237-01, Robinia pseudoacacia, F. I. S. (1, CNCI); Quebec: MRC Vaudreuil-Soulanges, Ville de l'Île-Perrot, 21.vi.2011 (14:00) beaten from Robinia pseudoacacia, P. de Tonnancour (2, CNCI; 1, CPTO); MRC Vaudreuil-Soulanges, Ville de l'̂̂le-Perrot, 1.vii.2011 (18:00) beaten from Robinia pseudoacacia, P. de Tonnancour (14, CNCI).

\section{Trichapion porcatum (Boheman, 1839), new to Quebec}

Note. This native species was recorded from Ontario by McNamara (1991) and from New Brunswick by Webster et al. (2012). 
Specimen data. Quebec: Gatineau, Queens Park, 9.vii.2011 (19:00) beaten from Desmodium canadense, P. de Tonnancour (2, CNCI; 1, CPTO); MRC VaudreuilSoulanges, Ville de l'Île-Perrot, $21 . v i .2011$ (14:00) beaten from Robinia pseudoacacia, P. de Tonnancour (1, CPTO).

\section{Subfamily Nanophyinae Gistel, 1848 \\ Tribe Nanophyini Gistel, 1848}

Nanophyes marmoratus marmoratus (Goeze, 1777), new to Ontario and Quebec

Note. This species was introduced to New York State for the biological control of purple loosestrife, Lythrum salicaria L. (Lythraceae) (Anderson 2003). It is likely that the new Ontario and Quebec records represent natural dispersal from the adjacent northern USA.

Specimen data. Ontario: Lanark Co., Packenham, 10.ix.2012, on Lythrum salicaria, E. St-Louis (1, CNCI); Quebec: MRC Vaudreuil-Soulanges, Saint-Lazare, 3.vi.2011, 1.ix.2011, swept or beaten from Lythrum salicaria, P. de Tonnancour (2, CPTO); MRC Vaudreuil-Soulanges, Notre-Dame-de-l'Île-Perrot, 19.v., 22.v., 30.v., 31.v.2011, 2.vi.2011, 2.vii.2011 (15:00), 29.viii.2011, 2.ix.2011, 1.vi.2012, all beaten or swept from Lythrum salicaria, P. de Tonnancour (18, CPTO; 23 CNCI); MRC Vaudreuil-Soulanges, Mt. Rigaud, 19.iv.2012, beaten from Salix sp., P. de Tonnancour (1, CPTO); MRC Les Collines-de-l'Outaouais, Bristol, Knox Landing Road, sand pit, 26.v.2012, on Lythrum salicaria, S. Laplante (2, CNCI).

\section{3) Family Dryophthoridae Schönherr, 1825 \\ Subfamily Rhynchophorinae Schönherr, 1833 \\ Tribe Sphenophorini Lacordaire, 1865}

\section{Sphenophorus incongruus Chittenden, 1905, new to Quebec}

Note. This native species was recorded from Ontario by McNamara (1991), and is associated with great rush, Schoenoplectus tabernaemontani (C.C.Gmel.) Palla, in wetlands (Vaurie 1951).

Specimen data. Quebec: MRC Deux-Montagnes, Oka, Parc national d'Oka, 7.iv.1991, under stone, lakeside, P. de Tonnancour (1, CPTO); MRC Deux-Montagnes, Oka, Parc Paul-Sauvé, 9.v.1993, 13.v.1994, P. Vigneault (2, CRVI). 


\section{4) Family Brachyceridae Billberg, 1820 \\ Subfamily Erirhininae Schönherr, 1825 \\ Tribe Stenopelmini LeConte, 1876}

\section{Lissorboptrus oryzophilus Kuschel, 1952, new to Quebec}

Note. This native species was previously known from Alberta (McNamara 1991). A pest of cultivated rice (where grown); larvae feed externally on roots (Anderson 2002). It is likely that related native semiaquatic grasses are the hosts elsewhere in North America.

Specimen data. Quebec: MRC Marguerite-D’Youville, Varennes (Verchères), 25.vi.2007, C. Chantal (1, CCCH).

\section{5) Family Curculionidae Latreille, 1802 Subfamily Curculioninae Latreille, 1802 Tribe Anthonomini Thomson, 1859}

\section{Anthonomus rufipes LeConte, 1876, new to Quebec}

Note. This native species was recorded from Alberta by McNamara (1991). Based on label data for specimens reported here, the species appears to be associated with Aster and Symphyotrichum spp. (Asteraceae).

Specimen data. Quebec: RCM Rouville, Rougemont, 4.vi.1966, C. Chantal (2, CCCH); MRC La Vallée-du-Richelieu, Saint-Lambert, 8.viii.1966, 16.vii.1967, P. de Tonnancour (3, CPTO); MRC L'Islet, Lac des Trois-Saumons, 1.vii.1968, C. Chantal (1, CCCH); Montreal, Dollard-des-Ormeaux, 25.viii.1974, C. Chantal (7, CCCH); MRC Vaudreuil-Soulanges, Terrasse-Vaudreuil, 17.vi.1993 (18:00), apical bud of Symphyotrichum novae-angliae, P. de Tonnancour (7, CPTO); MRC La Vallée-du-Richelieu, Mont-Saint-Hilaire, 9.vi.2004, H. Miquet-Sage (1, CHMS); MRC Vaudreuil-Soulanges, Notre-Dame-de-l'Île-Perrot, 31.v.2011, 1.vi., 2.vi., 17.vi.2011 (13:00, 14:00, 15:00), swept from Solidago/Aster, P. de Tonnancour (12, CPTO); MRC Vaudreuil-Soulanges, Ville de l'Île-Perrot, 4 June 2011 (11:00), swept from Symphyotrichum novae-angliae, P. de Tonnancour (3, CPTO); MRC Vaudreuil-Soulanges, Notre-Dame-de-l'Île-Perrot, 17.vi.2011 (13:00), swept from Aster sp., P. de Tonnancour (1, CPTO); MRC Vaudreuil-Soulanges, Ville de l'Île-Perrot, 13 August 2011 (13:00), swept from Aster sp., P. de Tonnancour (2, CPTO); MRC Vaudreuil-Soulanges, Notre-Dame-de-l'Île-Perrot, 1.vi.2012 (13:00), swept from Trifolium pratense, P. de Tonnancour (1, CPTO); MRC Vaudreuil-Soulanges, Saint-Lazare, 31.viii.2012, swept from Symphyotrichum novae-angliae, P. de Tonnancour (1, CPTO). 


\section{Anthonomus suturalis LeConte, 1824, new to Quebec}

Note. This species was recorded from British Columbia and Ontario by McNamara (1991). The species is associated with Phyloxera galls on leaves of Carya spp. (Juglandaceae) (Ahmad and Burke 1972).

Specimen data. Quebec: MRC Vaudreuil-Soulanges, Rigaud, 17.vii.1979, S. Laplante (1, CSLA); MRC Deux-Montagnes, Oka, Deux-Montagnes, beaten from Carya ovata, 13.vii., 15.vii.1982, 21.v.1983, C. Chantal (9, CCCH); Montreal, Dollard-des-Ormeaux, 16.vii.1982, C. Chantal (1, CCCH); Montreal, Sainte-Anne-deBellevue, 12.vi.1984, M.C. Larivière (3, LEMQ); Laval, Ste. Dorothée, 10.v.1987, F. Genier (1, CMNC); MRC Vaudreuil-Soulanges, Rigaud, 16.vi.1990, on Carya ovata, S. Laplante (1, CSLA); MRC Vaudreuil-Soulanges, Rigaud, 8.vii.1990 (15:00), beaten from Carya ovata, P. de Tonnancour (5, CPTO); Montreal, SainteAnne-de-Bellevue, 13.v.1992, S. Côté (1, CMNC); MRC Deux-Montagnes, Oka, 14.v.1993, 10.vii.1996, R. Vigneault (6, CRVI); MRC Deux-Montagnes, Oka, Parc d'Oka, Calvaire d'Oka, 5.vi.2004, R. Vigneault (1, CRVI); MRC VaudreuilSoulanges, Notre-Dame-de-l'Île-Perrot, $12 . v .2011$ (14:00), beaten from Rubus sp., P. de Tonnancour (1, CPTO); MRC Vaudreuil-Soulanges, Terrasse-Vaudreuil, 19.v.2011, 21.v.2011 (14:00, 23:00), beaten from Prunus nigra, white and UV light, P. de Tonnancour (2, CPTO).

\section{Anthonomus tectus LeConte, 1876, new to Alberta}

Note. This species is known in Canada only from the prairie provinces of Manitoba and Saskatchewan; we here add Alberta and document an association with Heterotheca villosa (Pursh.) Shinners (Asteraceae).

Specimen data. Alberta: $6.5 \mathrm{~km}$ E. Clyde, 15.vii.1989, swept from Heterotheca villosa (Pursh.) Shinners, R.S. Anderson (5, CMNC).

\section{Pseudanthonomus seriesetosus Dietz, 1891, new to New Brunswick}

Note. This eastern North American species is now recorded from New Brunswick. Adults have been associated with Vaccinium sp. (Ericaceae) (Clark 1987).

Specimen data. New Brunswick: Restigouche County, Dionne Brook P.N.A., $47.9030^{\circ} \mathrm{N}, 68.3503^{\circ} \mathrm{W}, 30 . v-15 . v i .2011$, Lindgren funnel trap, M. Roy \& V. Webster (1, CMNC; 1 , RWC). 


\section{Tribe Cionini Schönherr, 1825}

Cionus scrophulariae (Linnaeus, 1758), new to Canada

Note. This adventive Palaearctic species, which is associated with Scrophularia and Verbascum (Scrophulariaceae), is known to be established in New York (Anderson 2002).

Specimen data. Quebec: Montreal, 19.vi.2009, on Verbascum thapsus, CERL 15531, R. Limoges (1, CRLI).

\section{Tribe Curculionini Latreille, 1802}

\section{Curculio pardalis (Chittenden, 1908), new to Quebec}

Note. This native species was recorded in Canada from Manitoba and Ontario by McNamara (1991). It is is associated with Quercus spp. (Fagaceae) throughout its range (Gibson 1969).

Specimen data. Quebec: MRC Deux-Montagnes, Oka, Parc d'Oka, 6.vi.1995, R. Vigneault (1, CRVI); Parc Gatineau, Mont King, 2.vii.1995, R. Vigneault (2, CRVI); MRC Les Collines-de-l'Outaouais, Luskville, 18.vii.1996, R. Vigneault (1, CRVI); MRC Deux-Montagnes, Oka, 11.vi.1997, R. Vigneault (2, CRVI); MRC Vaudreuil-Soulanges, Rigaud, 12.vii.1997, UV Light, R. Vigneault (1, CRVI); MRC Les Collines-de-l'Outaouais, Eardley, 19.vii.1997, R. Vigneault (1, CRVI).

\section{Curculio sulcatulus (Casey, 1897), new to New Brunswick}

Note. This eastern North American species is associated with Quercus spp. throughout its range (Gibson 1969).

Specimen data. New Brunswick: Queens County, Jemseg, $45.8412^{\circ} \mathrm{N}, 66.1195^{\circ} \mathrm{W}$, 21.viii.-7.ix.2012, Lindgren trap in Quercus rubra canopy, C. Hughes \& K. Van Rooyen (1, RWC); Sunbury County, Sunpoke Lake, $45.7656^{\circ} \mathrm{N}, 66.5550^{\circ} \mathrm{W}, 15-27 . v i i i .2012$, Lindgren trap under Quercus rubra, C. Alderson \& V. Webster (1, RWC).

Tribe Mecinini Gistel, 1848

Mecinus janthinus (Germar, 1821), new to Quebec

Note. This adventive Palaearctic stem-mining weevil was introduced for the biological control of toadflaxes, Linaria spp. (Scrophulariaceae), and was known from Alberta, British Columbia, and Nova Scotia (Majka et al. 2007b, De Clerck-Floate and Cárcamo 2011). 
Specimen data. Quebec: MRC Marguerite-D'Youville, Varennes (Verchères), 30.vii.2011, 9.v., 12.v., 14.v., 24.v.2012, 1.vi.2012, C. Chantal (11, CCCH); MRC Pierre-De Saurel, Sorel-Tracy, 14.v.2012, C. Chantal (1, CCCH).

\section{Rhinusa neta (Germar, 1821), new to Quebec}

Note. This adventive Palaearctic species was introduced into British Columbia for control of toadflaxes, Linaria spp., (De Clerck-Floate and Cárcamo 2011).

Specimen data. Quebec: MRC Deux-Montagnes, Oka, Parc d'Oka, Calvaire d'Oka, 5.v.2000, R. Vigneault (1, CRVI).

\section{Tribe Rhamphini Rafinesque, 1815}

\section{Orchestes alni (Linnaeus, 1758), new to Ontario and Quebec}

Note. In Canada this species was previously only known from British Columbia. Adults and larvae of this adventive species are associated with Ulmus americana L. (Ulmaceae) (Anderson et al. 2007).

Specimen data. Ontario: Toronto, Yonge Street and York Mills Road, 30.v.2008, on Ulmus leaves, C. Grant (CFIA) (6, CNCI); Essex Co., Leamington, 17.v.2011, in greenhouse, Dean coll. (1, CNCI); Essex Co., Windsor, Malden Park, 17.iv.2012, Forestry Trapping, CFIA (1, CNCI); Quebec: MRC Le Haut-Saint-François, Scotstown, 16.vii.2007, C. Levesque (1, CNCI); Longueuil, 18.vi.2011 (18:00), swept from Ambrosia artemisiffolia, P. de Tonnancour (1, CPTO); Montreal, Sainte-Anne-de-Bellevue, 2.vii.2011 (13:00) beaten from Ulmus americana, P. de Tonnancour (10, CNCI; 14, CPTO); Montreal, SainteAnne-de-Bellevue, 16.vii.2011 (16:00), 24.viii.2011 (13:00), 31.viii.2011 (13:00), 12.ix.2011 (12:00), beaten from Ulmus americana (5) or U. parvifolia (4), P. de Tonnancour (16, CPTO).

\section{Tribe Smicronychini Seidlitz, 1891}

\section{Promecotarsus densus Casey, 1892, new to Alberta}

Note. This western North American prairie species is now recorded from Alberta. Nothing is known of the biology of this species.

Specimen data. Alberta: Division \#1, Onefour, 2.viii.1980, sweeping, G.A.P. Gibson (2, CMNC); Division \#1, Cypress Hills Interprovincial Park, 14.viii.1980, sweeping, G.A.P. Gibson (1, CMNC); Division \#1, C.F.B. Suffield, $50.628^{\circ} \mathrm{N}$, 110.306 $\mathrm{W}, 28 . v i i .1994$, A.T. Finnamore (6, CMNC). 


\section{Smicronyx griseus LeConte, 1876, new to Canada}

Note. This native species is distributed in the northeastern United States. Host plants are not known.

Specimen data. Ontario: Essex County, Windsor, Ojibway Prairie, 3-7.viii.2001, 12-13.ix.2002, M. Buck \& S. Paiero (2, CMNC).

\section{Smicronyx lineolatus Casey, 1892, new to Canada}

Note. This native species is distributed in the northeastern United States. Host plants are not known.

Specimen data. Manitoba: Junction Highways 21 and $38 \mathrm{~N}, 49.5626^{\circ} \mathrm{N}, 100.5299^{\circ} \mathrm{W}$, 7.vii.2007, tallgrass prairie, sweeping, R. Webster (2, RWC); Ontario: Haldimand-Norfolk Region, Delhi-Simcoe Railway Site, 11-14.vii.2001, yellow pans, S. Paiero (1, CMNC).

\section{Tribe Tychiini Gistel, 1848}

\section{Lignyodes bischoffi (Blatchley, 1916), new to New Brunswick}

Note. This native eastern North American species is associated with Fraxinus (Clark 1980).

Specimen data. New Brunswick: Sunbury County, Gilbert Island, $45.8770^{\circ} \mathrm{N}$, 66.2954 $4^{\circ} \mathrm{W}$, 25.vii-8.viii.2012, 8-21.viii.2012, Lindgren trap in Tilia americana canopy, C. Alderson, C. Hughes, \& V. Webster (3, RWC); Queens County, Jemseg, $45.8412^{\circ} \mathrm{N}, 66.1195^{\circ} \mathrm{W}, 8-21 . v i i i .2012$, Lindgren funnel trap, C. Alderson, C. Hughes, \& V. Webster (1, CNMC).

\section{Lignyodes horridulus (Casey, 1892), new to New Brunswick}

Note. This native central/eastern North American species is associated with Fraxinus (Clark 1980).

Specimen data. New Brunswick: York County, New Maryland, Charters Settlement, $45.8395^{\circ} \mathrm{N}, 66.7391^{\circ} \mathrm{W}, 18 . v i .2005$, UV light, R.P. Webster (1, RWC); Sunbury County, Gilbert Island, $45.8770^{\circ} \mathrm{N}, 66.2954^{\circ} \mathrm{W}, 28 . v-12 . v i .2012$, Lindgren funnel trap, C. Alderson, C. Hughes, \& V. Webster (1, CNMC).

\section{Tychius liljebladi Blatchley, 1916, new to Quebec}

Note. This widespread western and central native North American species is associated with Astragalus spp. (Fabaceae); larvae are in reproductive structures (Anderson 2002). 
In Canada the species was known from Alberta, Manitoba and Saskatchewan (McNamara 1991); these Quebec records are a significant eastward range extension.

Specimen data. Quebec: Gatineau, Aylmer, 18.vii.2004, 22.vii.2004, 28.vi.2005, 8.vii.2005, 9.vii.2011, on flowers of Astragalus canadensis, S. Laplante (25, CSLA, 10, CNCI, 2 CMNC); Gatineau, Aylmer, 28.vi.2005, F. Génier (8, CMNC); Gatineau, Aylmer, Queen's Park, 9.vii.2011, beaten from Astragalus canadensis, P. de Tonnancour (26, CPTO).

\section{Subfamily Bagoinae C.G. Thomson, 1859}

\section{Pnigodes setosus LeConte, 1876, new to Canada}

Note. This rarely collected native species has been recorded from the central United States north to Montana and South Dakota (O'Brien \& Wibmer 1982). It is associated with semi-aquatic habitats or wetlands.

Specimen data. Alberta: C.F.B. Suffield, $50.451^{\circ} \mathrm{N}, 110.762^{\circ} \mathrm{W}, 29 . v i .1994$, pan traps, A.T. Finnamore (3, CMNC).

\section{Subfamily Baridinae Schönherr, 1836 \\ Tribe Apostasimerini Schönherr, 1844}

\section{Barinus cribricollis (LeConte, 1876), new to Canada}

Note. This widespread native central USA species is recorded from Canada for the first time. Barinus species are associated with sedges in wetlands (Anderson 2002).

Specimen data. Quebec: RCM Pierre-De Saurel, Saint-Roch-de-Richelieu, 1.vi.2000, H. Miquet-Sage (3, CHMS); MRC Deux-Montagnes, Oka, Parc d'Oka, 28.v.2002, R. Vigneault (1, CMNC; 1, CRVI); MRC Marguerite-D’Youville, Varennes, 7.vi.2003, C. Chantal (1, CMNC); MRC Vaudreuil-Soulanges, Notre-Damede-l'Île-Perrot, 5.vi.2012, swept from Equisetum, Carex and grasses, P. de Tonnancour (1, CPTO); MRC Marguerite-D’Youville, Verchères, Contrecoeur, 7.vii.2012, swept from Carex, sandy bank of Saint-Lawrence River, H. Miquet-Sage (1, CPTO).

\section{Sibariops confinis (LeConte, 1876), new to Canada}

Note. This native eastern USA species is recorded from Canada for the first time. Sibariops species are associated with sedges in wetlands (Anderson 2002).

Specimen data. Quebec: Gatineau, Aylmer, 25.v.2012, swept from Cyperaceae, P. de Tonnancour (3, CPTO). 


\section{Sibariops confusus (Boheman, 1836), new to Canada}

Note. This widespread native eastern and central USA species is recorded from Canada for the first time. Sibariops species are associated with sedges in wetlands (Anderson 2002).

Specimen data. Quebec: MRC Le Haut-Richelieu, Saint-Blaise-sur-Richelieu, 19.iv.1980, C. Chantal (1, LEMQ); MRC La Vallée-du-Richelieu, Mont-Saint-Hilaire, 19.v.2004, H. Miquet-Sage (1, CHMS); MRC Vaudreuil-Soulanges, Notre-Dame-del'Île-Perrot, 5.vi.2012, swept from Scirpus and Eleocharis, P. de Tonnancour (1, CPTO).

\section{Tribe Baridini Schönherr, 1836}

\section{Cosmobaris scolopacea Germar, 1819, new to Manitoba}

Note. This adventive species is widespread in Canada. It is associated with various Chenopodiaceae (Ciegler 2010).

Specimen data. Manitoba: ca. $5 \mathrm{~km}$ E. Junction Highways $21 \& 345,49.3849^{\circ} \mathrm{N}$, $100.4378^{\circ} \mathrm{W}, 7 . v i i .2007$, sweeping, R.P. Webster (4, RWC).

\section{Trichobaris trinotata (Say, 1832), new to Quebec}

Note. This native species, the potato stalk borer, was previously known in Canada only from Ontario. This species is a pest of various Solanaceae; larvae feed in stems (Anderson 2002).

Specimen data. Quebec: MRC Deux-Montagnes, Oka, Parc d'Oka, 20.vi.2000 (1, CRVI).

\section{Tribe Madarini Jekel, 1865}

\section{Ampeloglypter ampelopsis (Riley, 1869), new to Quebec}

Note. This native species was previously known in Canada only from Ontario. This species is a pest of Vitis (grape; Vitaceae); larvae make galls on stems (Anderson 2002), often breaking the vine.

Specimen data. Quebec: MRC Vaudreuil-Soulanges, Terrasse-Vaudreuil, 19.v.2011, beaten from Prunus nigra, P. de Tonnancour (1, CPTO); MRC Vaudreuil-Soulanges, Notre-Dame-de-l'Île-Perrot, 20 May 2011 (17:00), beaten from Spiraea $x$ vanhouttei \& Rubus odoratus, P. de Tonnancour (2, CPTO); Montreal, SteAnne-de-Bellevue, 28.vi.2011, beaten from leaves of Juglans nigra, P. de Tonnancour (1, CPTO). 


\section{Desmoglyptus crenatus (LeConte, 1876), new to Canada}

Note. This rare, native species is known from the northeastern USA: District of Columbia, Maryland, Pennsylvania, and Virginia, and occurs on wild grape, Vitis sp. (Vitaceae) (Blatchley and Leng 1916).

Specimen data. Ontario: Essex County, Point Pelee National Park, 11-17 Jul 2003, yellow pan traps in Opuntia sp. field , D. Cheung, (1, CMNC), debu00219744.

\section{Subfamily Ceutorhynchinae Gistel, 1848}

Tribe Ceutorhynchini Gistel, 1848

\section{Ceutorhynchus hamiltoni Dietz, 1896, new to Quebec}

Note. This native species is widespread along the eastern coastal USA and maritime provinces of Canada (Majka et al. 2007b) on American searocket, Cakile edentula Bigelow (Hook.) (Brassicaceae).

Specimen data. Quebec: RCM La Haute-Gaspésie, Cap Chat, 21.vii.1954, on Cakile edentula, J.E.H. Martin (2, CMNC; 47, CNCI); RCM Bonaventure, New Richmond, 6.viii.1954, on Cakile edentula, J.E.H. Martin (5, CNCI).

\section{Microplontus campestris (Gyllenhal, 1837), new to Quebec}

Note. This adventive Palaearctic species is associated with Leucanthemum vulgare (L.) (Asteraceae) and may help control this invasive weed. This weevil was accidentally introduced into North America, and has been present in Ontario since 1971, or earlier (Anderson and Korotyaev 2004).

Specimen data. Quebec: MRC Marguerite-D’Youville, Varennes (Verchères), 30.vi.2008, C. Chantal (1, CCCH).

\section{Tribe Cnemogonini Colonnelli, 1979}

\section{Dietzella zimmermanni (Gyllenhal, 1837), new to New Brunswick}

Note. This transcontinental native species is recorded from New Brunswick for the first time. It is associated with Epilobium (Ciegler 2010).

Specimen data. New Brunswick: Restigouche County, Jacquet River Gorge P.N.A., $47.7491^{\circ} \mathrm{N}, 66.1114^{\circ} \mathrm{W}, 24 . v i .2008$, R.P. Webster, swept from foliage (1, RWC). 


\section{Tribe Phytobiini Gistel, 1848}

\section{Parenthis vestitus Dietz, 1896, new to New Brunswick}

Note. This native eastern North American species was previously known in Canada only from Ontario. It is associated with wetlands.

Specimen data. New Brunswick: Queens Co., Jemseg, $45.8412^{\circ} \mathrm{N}, 66.1195^{\circ} \mathrm{W}$, 25.vii-8.viii.2012, C. Alderson, C. Hughes, \& V. Webster, Lindgren trap (1, RWC); Sunbury County, Gilbert Island, $45.8770^{\circ} \mathrm{N}, 66.2954^{\circ} \mathrm{W}, 18-28 . V .2012$, 25.V-12.vi.2012, 11-25.vii.2012, Lindgren trap under Juglans cinerea, C. Alderson, C. Hughes \& V. Webster (1, CMNC; 5, RWC); Sunbury County, Gilbert Island, $45.8770^{\circ} \mathrm{N}, 66.2954^{\circ} \mathrm{W}, 21 . v i i i-7 . i x .2012$, C. Hughes \& K. Van Rooyen (1, CMNC).

\section{Pelenomus squamosus LeConte, 1876, new to New Brunswick}

Note. This transcontinental North American species is recorded from the Maritime Provinces for the first time. It is associated with wetlands.

Specimen data. New Brunswick: Queens County, Jemseg, $45.8412^{\circ} \mathrm{N}$, 66.1195 ${ }^{\circ} \mathrm{W}, 14-28 . v .2012$, Lindgren funnel, C. Alderson, C. Hughes, \& V. Webster (1, RWC); Restigouche County, Wild Goose Lake, $420 \mathrm{~m}$ elev., $47.8540^{\circ} \mathrm{N}$, 66.3219 ${ }^{\circ} \mathrm{W}, 7 . v i .2012$, treading Carex \& grasses, R. Webster \& M. Turgeon (1, RWC); Sunbury County, Gilbert Island, $45.8770^{\circ} \mathrm{N}, 66.2954^{\circ} \mathrm{W}, 11-25 . v i i .2012$, Lindgren trap, C. Alderson, C. Hughes \& V. Webster (1, CMNC).

Tribe Scleropterini Schultze, 1902

Asperosoma echinatum (Fall, 1917), new to Ontario

Note. This species (Fig. 1) is associated with the native grassland forb Heuchera richardsoni R. Br. (Saxifragiaceae) (Fall 1917) and was previously known only from Manitoba (McNamara 1991). This species is at present a Canadian endemic, although it may also exist in USA. Targeted collecting efforts at other Ontario sites have not yielded additional specimens.

Specimen data. Ontario: Essex Co., Windsor, Burnt Prairie, v.2001, S. Paiero, CNC COLEOPT \#04-5422 (1, CMNC). 


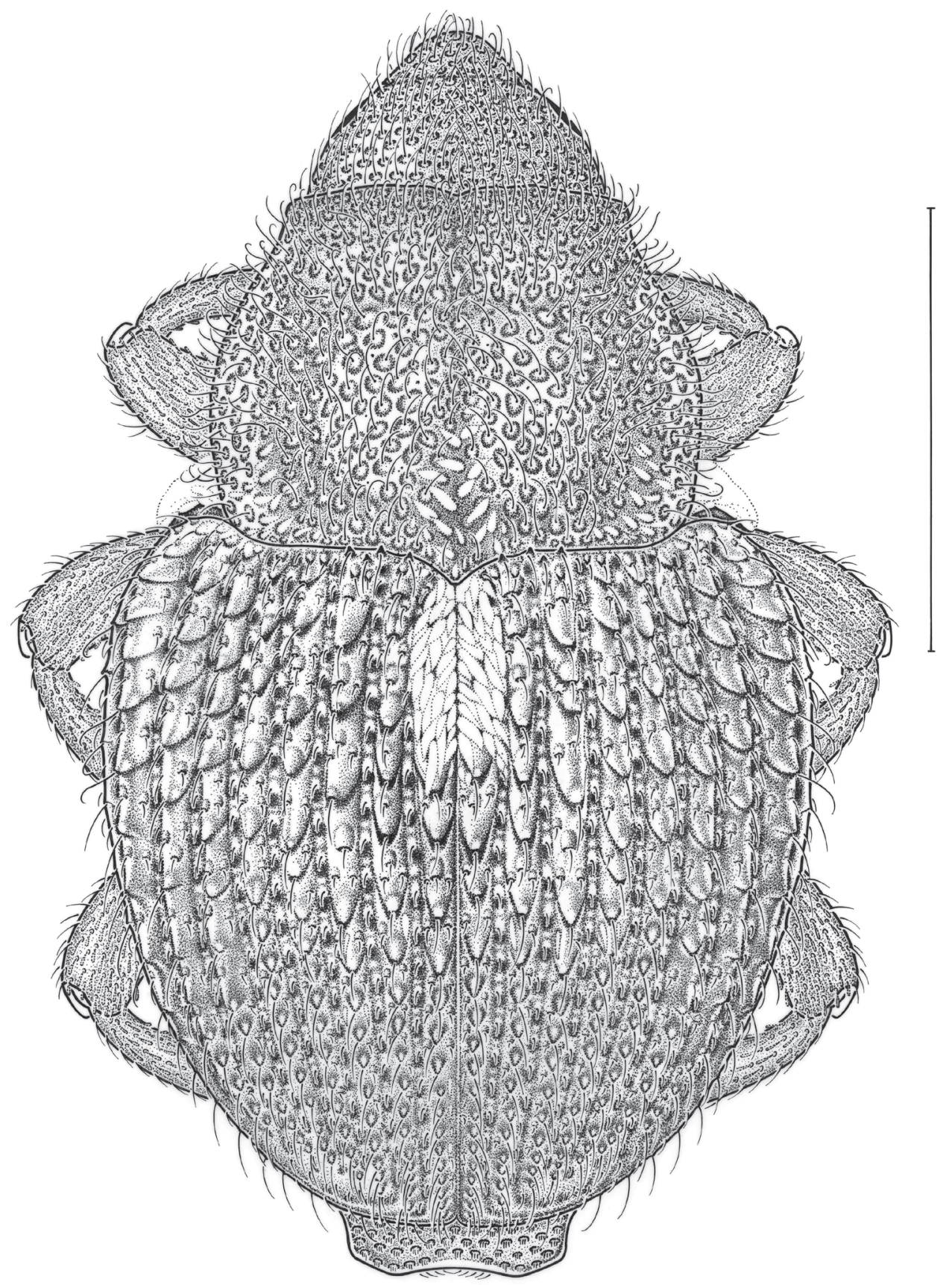

Figure I. Dorsal habitus drawing of Asperosoma echinatum (Fall, 1917), a species to-date only known from Canada. Scale line $=1 \mathrm{~mm}$. 


\section{Subfamily Conoderinae Schönherr, 1833}

Tribe Lechriopini Lacordaire, 1865

\section{Psomus armatus Dietz, 1891, new to New Brunswick}

Note. This native eastern North American species is recorded from the Maritime Provinces for the first time. It is associated with forest habitats, and has been recorded on sprouts of white ash, Fraxinus americanus L. (Blatchley \& Leng 1916).

Specimen data. New Brunswick: Carleton County, Jackson Falls, Bell Forest, $46.2200^{\circ} \mathrm{N},-67.7231^{\circ} \mathrm{W}, 3-17 . v i i .2012$, Lindgren trap in Tilia americana canopy, C. Alderson \& V. Webster (1, RWC); Queens County, Jemseg, $45.8412^{\circ} \mathrm{N}, 66.1195^{\circ} \mathrm{W}$, 28.vi-10.vii.2012, Lindgren funnel trap, C. Alderson \&V. Webster (1, RWC).

\section{Tribe Zygopini Lacordaire, 1865}

\section{Cylindrocopturus furnissi Buchanan, 1940, new to Canada}

Note. This native species, known in USA as the Douglas-fir twig weevil, was recorded from California, Oregon and Washington by O'Brien and Wibmer (1982). It is a minor pest of shoots of weakened Pseudotsuga spp. (Pinaceae).

Specimen data. British Columbia: Kootenays Region, Wynndel (2 mi. N.), 28.vi.-3.vii.1980, R. Anderson (1, CMNC).

\section{Cylindrocopturus quercus (Say, 1832), new to Canada}

Note. This native species was recorded from eastern USA by O'Brien and Wibmer (1982). Adults of this species breed in ragweed, Ambrosia artemesiifolia L. (Piper 1977).

Specimen data. Quebec: Montreal, (1, CNCI); Ormstown, 29.vi.1978, E.J. Kiteley (1, CNCI); MRC Vaudreuil-Soulanges, Notre-Dame-de-l'Île-Perrot, 19.vi.2011 (17:00), 12.viii.2012 (17:00), swept from Erigeron sp., beaten from Asclepias syriaca, P. de Tonnancour (3, CPTO).

\section{Subfamily Cossoninae Schönherr, 1825 Tribe Dryotribini LeConte, 1876 \\ Caulophilus dubius (Horn, 1873), new to Canada}

Note. This native species was recorded from eastern USA north to Illinois, Michigan, New York, Ohio and Pennsylvania by O'Brien and Wibmer (1982). Adults are found beneath the dead tree bark (Blatchley and Leng 1916). 
Specimen data. Quebec: Montreal, Sainte-Anne-de-Bellevue, Morgan Arboretum, yellow pan traps, 25-29.vi.2001, J. Forrest (1, LEMQ).

\section{Tribe Onycholipini Wollaston, 1873}

Pseudopentarthrum parvicollis (Casey, 1892), new to Canada

Note. This native species is widespread in the eastern USA from TX north to NY. It is associated with decaying wood, particularly old tree holes.

Specimen data. Ontario: Kent County, Rondeau Provincial Park, Spicebush Trail, 42 $18^{\prime} 09^{\prime \prime N}, 81^{\circ} 51^{\prime} 06 " \mathrm{~W}, 16-29$ Jul 2003, S.A. Marshall, Malaise Trap in Carolinian forest (1; CMNC), debu01123692.

\section{Tribe Rhyncolini Gistel, 1848}

\section{Rhyncolus knowltoni (Thatcher, 1940), new to Alberta, and Saskatchewan}

Note. This native western North American species was previously only known in Canada from Manitoba. It has been associated with Populus tremuloides Michx.

Specimen data. Alberta: Southern Alberta, Medicine Hat, viii.1980, pan trap, G.A.P. Gibson, (1; CMNC); Division \#2, 0.5 km E. Writing-on-Stone Provincial Park, 6-15.vi.1981, pan traps, D. McCorquodale (1; CMNC); Division \#1, Cypress Hills Interprovincial Park, 4 km S. Elkwater, 22.vi-19.viii.1988, 1400m, S. \& J. Peck, fir-pine-aspen forest, FIT (1; CMNC); Saskatchewan: Maple Creek No. 111, Cypress Hills Interprovincial Park, Center Block, Boiler Creek aspen forest, 21.vi-19.viii.1988, FIT $1200 \mathrm{~m}$, S. \& J. Peck (1; CMNC).

\section{Rhyncolus macrops Buchanan, 1946, new to New Brunswick}

Note. This transcontinental native North American species is recorded from New Brunswick for the first time. Adults are associated with a variety of dead coniferous trees (Pinales) (Buchanan 1946).

Specimen data. New Brunswick: Carleton County, Jackson Falls, Bell Forest, $46.2200^{\circ} \mathrm{N}, 67.7231^{\circ} \mathrm{W}, 7 . v i .2007,27 . v i .2008$ under spruce bark \& in Lindgren trap, R. Webster (2, RWC); Sunbury County, Acadia Research Forest, $45.9866^{\circ} \mathrm{N}, 66.3841^{\circ} \mathrm{W}, 19-25 . v .2009 ， 25 . v-2 . v i .2009 ， 16-24 . v i .2009$, red spruce forest, Lindgren traps, R. Webster \& M.A. Giguère (3, RWC); York County, Charters Settlement, $45.8380^{\circ} \mathrm{N}, 66.7300^{\circ} \mathrm{W}, 6 . v .2004$, under bark, R. Webster (1, RWC). 


\section{Subfamily Cryptorhynchinae Schönherr, 1825 \\ Tribe Cryptorhynchini Schönherr, 1825}

\section{Acalles carinatus LeConte, 1876, new to Quebec}

Note. Label data from many specimens from throughout its range in eastern USA and southern Ontario indicates that this native species occurs commonly in hardwood forest leaf litter.

Specimen data. Quebec: MRC Deux-Montagnes, Oka, Parc d'Oka, 11.vi.1995, R. Vigneault (1, CRVI); MRC La Vallée-du-Richelieu, Mont-Saint-Hilaire, yellow pan traps, 11-18.vi.2001, E. Fast (1, CMNC); MRC La Vallée-du-Richelieu, MontSaint-Hilaire, beach-sugar maple forest, yellow pan traps, 21-28.v.2001, E. Fast (1, LEMQ); same except: 16-23.vii.2001 (1, LEMQ); Montreal, Sainte-Anne-de-Bellevue, Morgan Arboretum, 10-15.vi., 15-20.vi., 20-25.vi., 3-9.vii., 20-26.viii.2001, J. Forrest, yellow pan traps in ash-sugar maple forest (6, LEMQ).

\section{Cryptorhynchus tristis LeConte, 1876, new to Canada}

Note. This widespread native eastern USA species is said to feed on leaves of Quercus coccinea Wang. (Fagaceae, scarlet oak) at night and larvae develop under the bark (Anderson 2008).

Specimen data. Quebec: RCM Brome-Missisquoi, Saint-Armand, 12.vii.2008, P. Bélanger, UV light (1, LEMQ).

\section{Tyloderma foveolatum (Say, 1832), new to Manitoba}

Note. This widespread native eastern USA and eastern southern Canadian species has been associated with Oenothera biennis L. (Onagraceae), a native ruderal plant (Wibmer 1981).

Specimen data. Manitoba: Eastern Manitoba, Sandilands Provincial Forest, Marchand (10-12 km E.), 10-12.vi.1987, H. \& A. Howden (1, CMNC); Spruce Woods Provincial Park, Glenboro (10-15 km W.), 17.vi.1987, H. \& A. Howden (3, CMNC).

\section{Subfamily Cyclominae Schönherr, 1826}

Tribe Listroderini LeConte, 1876

\section{Listronotus maculicollis (Kirby, 1837), new to Manitoba}

Note. This transcontinental North American native species is recorded from Manitoba for the first time. It is associated with wetlands.

Specimen data. Manitoba: Aweme at Assiniboine River, $49.673^{\circ} \mathrm{N}, 99.565^{\circ} \mathrm{W}$, 11.vii.2003, R.P. Webster (1, RWC). 


\section{Listronotus punctiger LeConte, 1876, new to Manitoba}

Note. This western North Americans native pecies is recorded from Manitoba for the first time. It is associated with wetlands.

Specimen data. Manitoba: near Junction Highways $21 \& 543 \mathrm{~N}, 49.6705^{\circ} \mathrm{N}$, $100.4646^{\circ} \mathrm{W}, 6 . v i i .2007$, sweeping, R.P. Webster (1, RWC).

\section{Subfamily Entiminae Schönherr, 1823 \\ Tribe Cyphicerini Lacordaire, 1863 \\ Cyrtepistomus castaneus (Roelofs, 1873), new to Quebec}

Note. This adventive Palaearctic species is widespread in the eastern USA into Ontario and is known in USA as the Asiatic oak weevil; it can be extremely common locally (Anderson 2002), and acts as a minor defoliator of broadleaved trees.

Specimen data. Quebec: RCM La Côte-de-Gaspé, Gaspé, 27.viii.1966, W. Boyle (1, LEMQ).

\section{Tribe Naupactini Gistel, 1848}

\section{Atrichonotus taeniatulus (Berg, 1881), new to Canada}

Note. This adventive species, the adults of which feed on the roots and foliage of a variety of host plants (although most frequently on Fabaceae), was known previously in North America from southeastern USA west to Texas (Anderson 2002). This species can be an important pest of alfalfa (Medicago sativa $\mathrm{L}$., Fabaceae).

Specimen data. Quebec: MRC La Vallée-du-Richelieu, Mont-Saint-Hilaire, 25.v.1999, H. Miquet-Sage (1, CHMS).

\section{Tribe Otiorhynchini Schönherr, 1826}

\section{Otiorhynchus ligustici (Linnaeus, 1758), new to Quebec}

Note. This adventive Palaearctic species was known in Canada only from Ontario (Bright and Bouchard 2008). Also known as the alfalfa snout beetle this species is a major pest of alfalfa.

Specimen data. Quebec: MRC Beauharnois-Salaberry, Salaberry-de-Valleyfield, 13.vi.2012, on grasses under Salix, Populus and Vitis riparia, Y. Racine (1, CPTO). 


\section{Subfamily Lixinae Schönherr, 1823}

Tribe Lixini Schönherr, 1823

\section{Larinus planus (Fabricius, 1792), new to Quebec}

Note. This adventive Palaearctic species was introduced to North America for the biological control of Canada thistle, Cirsium arvense (L.) Scop. (Asteraceae) (Anderson 2002). It is present in Alberta, British Columbia, Nova Scotia and Ontario.

Specimen data. Quebec: MRC Marguerite-D'Youville, Varennes (Verchères), 25.viii.2001, 6.ix.2001, 16.viii.2004, 12.vi.2006, 13.vi.2006, C. Chantal (8, CCCH); MRC Vaudreuil-Soulanges, Notre-Dame-de-l'Île-Perrot, 20.vi.2009, 21.vi.2009, 30.viii.2009, 30.viii.2012, all on Cirsium arvense, P. de Tonnancour (6, CPTO); Montreal, Sainte-Anne-de-Bellevue, 25.vi.2010, 7.vi., 11.vi.2011, on Cirsium arvense, P. de Tonnancour (12, CPTO).

\section{Subfamily Mesoptiliinae Lacordaire, 1863}

Tribe Magdalidini Pascoe, 1870

\section{Magdalis inconspicua Horn, 1873, new to New Brunswick}

Note. This native eastern North American species is recorded from the Maritime Provinces for the first time. It is associated with forest habitats.

Specimen data. New Brunswick: Sunbury County, Gilbert Island, $45.8770^{\circ} \mathrm{N}$, $66.2954^{\circ} \mathrm{W}, 29 . v i .-11 . v i i .2012$, Lindgren traps in canopy of Juglans cinerea \& Tilia americana, and under Tilia americana, C. Alderson \& V. Webster (2, AFC; 1, CMNC; 1, NBM; 7, RWC); Carleton County, Jackson Falls, Bell Forest, $46.2200^{\circ} \mathrm{N}$, $67.7231^{\circ} \mathrm{W}, 7-21 . v i .2012$, Lindgren traps in Tilia americana canopy, C. Alderson \& V. Webster (1, CMNC; 1, RWC).

\section{Magdalis salicis Horn, 1873, new to New Brunswick}

Note. This native eastern North American species is recorded from New Brunswick for the first time. It is associated with forest habitats.

Specimen data. New Brunswick: Queens County, Grand Lake Meadows PNA, $45.8227^{\circ} \mathrm{N}, 66.1209^{\circ} \mathrm{W}, 21 . v i-5 . v i i .2011$, Lindgren trap in canopy, M. Roy \& V. Webster (1, RWC); Sunbury County, Gilbert Island, $45.8770^{\circ} \mathrm{N}, 66.2954^{\circ} \mathrm{W}$, 12.vii.2012, sweeping, R.P. Webster (1, RWC). 


\section{Subfamily Molytinae Schönherr, 1823 \\ Tribe Conotrachelini Jekel, 1865}

\section{Microbyus setiger LeConte, 1876, new to Quebec}

Note. Adults of this widespread native eastern USA (and into Ontario) species have been associated with dead Fagus (Fagaceae, beech) (Anderson 2002).

Specimen data. Quebec: MRC Deux-Montagnes, Oka, Parc d'Oka, 30.v.1995, R. Vigneault (1, CRVI); Brome-Missisquoi, Saint-Armand, 9.vi.2003, Claude Chantal (1, CCCH); MRC Marguerite-D’Youville, Varennes (Verchères), 6.vi.2011, C. Chantal $(1, \mathrm{CCCH})$.

\section{Subfamily Scolytinae Latreille, 1804}

The Scolytinae, or bark beetles are a distinctive and relatively well-known subfamily that includes many forest pests. Scolytinae have been a focus of adventive forest pest trapping surveys by the Canadian Food Inspection Agency, the Canadian Forest Service and others. The taxonomy and distribution of species that are not readily captured in traps, or attack smaller diameter stems remain less well-known.

\section{Tribe Dryocoetini Lindemann, 1877}

\section{Dryocoetes autographus (Ratzeburg, 1837), new to Nova Scotia}

Note. This widespread native species, known from all other provinces and two territories, attacks the lower parts of dead and dying conifers. The absence of records from Nova Scotia seems to be an oversight.

Specimen data. Nova Scotia: Colchester Co., Portapique, $45.405^{\circ} \mathrm{N}, 63.704^{\circ} \mathrm{W}$, 26.vii.1927, C.A. Frost (1, CNCI), CNC Diptera 126927; Kejimkujik National Park, $44.386^{\circ} \mathrm{N}, 65.293^{\circ} \mathrm{W}, 17 . v i i .1970$, ex. Picea glauca, D.E. Bright (2, CNCI), CNC Diptera 127810, 127811; St. Ann's Gut, 46.217º N, 60.616 $\mathrm{W}, 3 . v i i i .1970$, ex. Picea, D.E. Bright (2, CNCI), CNC Diptera 127830, 127831 ; White point, $46.883^{\circ} \mathrm{N}$, 60.363 W, 23.vi.1983, Y. Bousquet (1, CNCI), CNC Diptera 128020, 128021, 128022; Cape Breton Highlands National Park, Lone Shieling, $46.897^{\circ} \mathrm{N}, 60.783^{\circ} \mathrm{W}$, 1.vii.1983, R. Vockeroth, L. Lesage, Y. Bousquet (2, CNCI), CNC Diptera 128037, 128038, 128072; Cape Breton Highlands National Park, Jack Pine Trail, $46.779^{\circ} \mathrm{N}$, 60.333W, 22.vii.1983, D.E. \& J.E. Bright (1, CNCI), CNC Diptera 128028; Halifax, Point Pleasant Park, 46.822 ${ }^{\circ} \mathrm{N}, 60.799^{\circ} \mathrm{W}, 13 . v i .-5 . v i i .1990$, S. Robertson \& G Harding (4, CNCI), CNC Diptera 127944, 127945, 127946, 127947. 


\section{Dryocoetes caryi Hopkins, 1915, new to Prince Edward Island}

Note. This rarely collected native species typically inhabits small, stressed Picea spp. (Pinaceae) trees and is known from across Canada (Alberta, British Columbia, New Brunswick, Nova Scotia, and Quebec) Bright 1976, Webster et al. 2012).

Specimen data. Prince Edward Island, Queens Co., Charlottetown, 23.vi.-7. vii.2000, funnel trap, CFIA (1, CNCI).

\section{Lymantor alaskanus Wood, 1978, new to Canada}

Note. This native species was only known from the type series, collected in 1978 near Fairbanks Alaska. The Alberta specimens mentioned here represent a significant extension of the known range to the south and east. Both the type series and all specimens reported here were captured in CFIA traps baited with ipsenol lure.

Specimen data. Alberta: RM (Regional Municipality) of Wood Buffalo, $56.733^{\circ} \mathrm{N}$, $111.384^{\circ} \mathrm{W}, 7 . v i i .2005$, funnel trap with ipsenol, CFIA (3, CNCI), CNC COLEO 00106296, 00106297; RM of Wood Buffalo, $56.733^{\circ} \mathrm{N}, 111.384^{\circ} \mathrm{W}, 28 . i v .-29$. ix.2005, funnel trap (2, CFIA), CNCI, CNC COLEO 00106298.

\section{Tribe Hylastini LeConte, 1876}

\section{Hylastes macer LeConte, 1868, new to Alberta}

Note. This western species feeds mainly on Pinus spp. (Pinaceae), and was already known from nearby parts of British Columbia (Bright 1976).

Specimen data. Alberta: Calgary, $51.042^{\circ} \mathrm{N}, 114.078^{\circ} \mathrm{W}, 11 . v i .1944$, in flight, E.J. Kitely (1, CNCI), CNC Diptera 129000.

\section{Hylastes opacus Erichson, 1836, new data on first Canadian and Quebec records}

Note. These records of this adventive, Pinus-feeding Palaearctic species (also known from New Brunswick, Webster et al. 2012) were reported by Bright and Skidmore (1997) without specimen data.

Specimen data. Ontario: Elgin Co., Port Bruce, $42.650^{\circ} \mathrm{N}, 81.017^{\circ} \mathrm{W}$, 19.iv.1995, J. Hale (2, CNCI), CNC COLEO 00105928, 00105929; Quebec: Montreal, $45.500^{\circ} \mathrm{N}, 73.600^{\circ} \mathrm{W}$, 6.vi.1997, D. Couture (2, CNCI), CNC COLEO $00105926,00105927$. 


\section{Tribe Hylurgini Gistel, 1848}

\section{Dendroctonus ponderosae Hopkins, 1902, new to Alberta}

Note. The native, Pinus-feeding mountain pine beetle has not previously been reported from Alberta in the taxonomic literature despite well-studied, costly outbreaks there. Specimens listed here document the oldest CNCI material from Canada outside British Columbia.

Specimen data. Alberta: Waterton Lakes National Park, Summit-Carthew Lakes Trail, $49.033^{\circ} \mathrm{N}, 113.984^{\circ} \mathrm{W}, 17 . v i .1980$, J.M Campbell \& D.E. Bright (6, CNCI), CNC Diptera 130912-130917; Waterton Lakes National Park, km 3 Chief Mountain Highway, 17.vi.1980, J.M. Campbell (1, CNCI), CNC Diptera 130911; Waterton Lakes National Park, Red Rock Canyon, $49.133^{\circ} \mathrm{N}, 113.018^{\circ} \mathrm{W}$, 16.vii.1980, ex. Pinus contorta, D.E. Bright (1, CNCI), CNC Diptera 130910; Waterton Lakes National Park, km 9 Chief Mountain Highway, 24.vii.1980, ex. Pinus contorta, D.E. Bright (3, CNCI), CNC Diptera 130918-130920; Waterton Lakes National Park, Cameron Lake, $49.017^{\circ} \mathrm{N}, 114.067^{\circ} \mathrm{W}$, 30.vii.1980, D.E. Bright (4, CNCI), CNC Diptera 130902-130905; Waterton Lakes National Park, Belly River, $49.767^{\circ} \mathrm{N}, 113.034^{\circ} \mathrm{W}, 30 . v i i .1980$, D.E. Bright (4, CNCI), CNC Diptera 130906-130909.

\section{Dendroctonus simplex LeConte, 1868, new to Yukon and Nunavut: not known from Northwest Territories}

Note. The native eastern larch beetle is reported from all ten provinces, and Northwest Territories (Bright 1976). With the separation of Nunavut from Northwest Territories, the single Northwest Territories record should become a Nunavut record.

Specimen data. Nunavut: Keewatin, Padlei, $61.933^{\circ} \mathrm{N}, 96.650^{\circ} \mathrm{W}, 27 . v i i .1950$, R.E. Duckworth (1, CNCI), CNC COLEO 00100743; Yukon: Km 382, Dempster Highway, $66.386^{\circ} \mathrm{N}, 136.317^{\circ} \mathrm{W}, 23 . v i .1981$, D.E. Bright (1, CNCI), CNC Diptera 132434.

Tomicus piniperda (Linnaeus, 1758), new data on first Canadian record, and first Quebec record

Note. These records of this adventive Palaearctic species, the pine shoot beetle, were reported by Bright and Skidmore (1997) without specimen data.

Specimen data. Ontario: Haldimand Co., Dunnville, $42.904^{\circ} \mathrm{N}, 79.618^{\circ} \mathrm{W}$, iv.1993, Agriculture Canada (8, CNCI), CNC Diptera 128768 to 128775; Haldimand Co., Dunnville, $42.904^{\circ} \mathrm{N}, 79.618^{\circ} \mathrm{W}, 23 . v i .1993$, ex. bole of Pinus sylvestris, D.E. Bright (12, CNCI), CNC Diptera 128776 to 128782; Quebec: Gatineau, Aylmer, $45.400^{\circ} \mathrm{N}, 75.817^{\circ} \mathrm{W}$, 9.vii.1993, ex. bole Pinus sylvestris, D.E. Bright (1, CNCI), CNC Diptera 128783. 


\section{Tribe Ipini Bedel, 1888}

\section{Ips perroti Swaine, 1915, new to Nova Scotia}

Note. This native species breeds in thin-barked Pinus spp. stems, and is known from Alberta, British Columbia, Manitoba, New Brunswick, Ontario, Quebec, and Saskatchewan.

Specimen data. Nova Scotia: Cape Breton, Bras d'Or, $46.250^{\circ} \mathrm{N}, 60.282^{\circ} \mathrm{W}$, 1-21.vi.2000, ex. funnel trap, CFIA (1, CNCI), CNC COLEO 00105995.

\section{Tribe Micracidini LeConte, 1876}

\section{Hylocurus rudis (LeConte, 1876), new to Canada}

Note. This native species breeds in weakened or dead small diameter stems of hardwood trees. Its apparent limitation to southern Ontario and Quebec is probably due to climate, given that it is also known from Michigan, Ohio and Pennsylvania (Wood and Bright 1992).

Specimen data. Ontario: Essex Co., Pt. Pelee National Park, Visitor's Centre, 22-29.v.2000, O. Lonsdale (1, DEBU), debu01000657; Essex Co., Middle Island, $40^{\circ} 41^{\prime} \mathrm{N}, 82^{\circ} 41^{\prime} \mathrm{W}, 4 . v i i .2000$, ex. yellow pans etc., Paiero, Marshall, \& Cheung (4, DEBU), debu00221910, debu00222016, debu00221976, debu00221526; Essex Co., Middle Island, $40^{\circ} 41^{\prime} \mathrm{N}, 82^{\circ} 41^{\prime} \mathrm{W}, 11 . v i .2003$, S.A. Marshall (1, DEBU), debu00221012; Essex Co., Pt. Pelee National Park, The Tip, 17.vi.2003, H. Carscadden (1, DEBU), debu00219553; Halton, Oakville, $43.450^{\circ} \mathrm{N}, 79.683^{\circ} \mathrm{W}, 9-23$. vi.2008, ex. funnel trap, CFIA (1, CNCI), CNC COLEO 00105968; Quebec: MRC Deux-Montagnes, Oka, Parc d'Oka, Deux-Montagnes, 27.iv.1997, reared ex. Carya ovata R. Vigneault, (1, CRVI).

\section{Micracis suturalis LeConte, 1868 , new to Ontario}

Note. This native species breeds in Cercis spp. (Fabaceae), Juglans spp. (Juglandaceae), and other broadleaved trees (Bright 1976), and is already known from Quebec (Chantal 1992).

Specimen data. Ontario: Kent Co., Rondeau Provincial Park, 1.vi.1982, D.E. Bright (1, CNCI), CNC COLEO 00155768.

\section{Pseudothysanoes rigidus (LeConte, 1876), new data on first Ontario record}

Note. This native species, known from USA, breeds in Tilia sp. (Malvaceae), and has been previously reported from Ontario without additional data (Bright 1976). 
Specimen data. Ontario: RM Halton, Burlington, Sheldon Creek Woodlot, $43.396^{\circ} \mathrm{N}, 79.775^{\circ} \mathrm{W}, 1-14 . v i .2007$, ex. funnel trap, CFIA (1, CNCI), CNC COLEO 00106106.

\section{Tribe Phloeosinini Nüsslin, 1912}

\section{Phloeosinus pini Swaine, 1915, new to Ontario}

Note. This widespread (Alberta, British Columbia, Manitoba, Quebec, Northwest Territories, Nova Scotia, Yukon), but infrequently collected native species breeds in Pinus and Picea (Bright 1976).

Specimen data. Ontario: Lennox and Addington Co., Napanee, $44.267^{\circ} \mathrm{N}$, $76.971^{\circ} \mathrm{W}, 20 . v .2004$, ex. funnel trap, CFIA (1, CNCI).

\section{Tribe Phloeotribini Chapuis, 1869}

\section{Phloeotribus liminaris (Harris, 1852), new to Saskatchewan}

Note. The peach bark beetle breeds in Prunus spp., is native and already known from neighbouring Manitoba, and also Ontario, Quebec, New Brunswick, and Nova Scotia (Bright 1976, Majka et al. 2007).

Specimen data. Saskatchewan: Saskatoon, Avenue K. S., vi.2011. J. Boone (1, City of Saskatoon).

\section{Phloetribus piceae Swaine, 1911, new to Yukon}

Note. This infrequently collected boreal species breeds in Picea spp (Bright 1976), and was known from British Columbia, Manitoba, Ontario, Quebec, New Brunswick, Nova Scotia, and Northwest Territories.

Specimen data. Yukon: $\mathrm{Km} 72$, Dempster Highway, $64.53^{\circ} \mathrm{N}, 138.231^{\circ} \mathrm{W}$, 20.vi.1981, ex. Picea glauca, D.E. Bright (6, CNCI), CNC COLEO 00104642, CNC Diptera 130333-130336.

\section{Phloeotribus scabricollis (Hopkins, 1916), new to Canada}

Note. This rarely collected species breeds in Ptelea trifoliata L. (Rutaceae) and Staphylea trifolia L. (Staphyleaceae) (Wood 1982).

Specimen data. Ontario: Essex Co., Pelee Island, 11.vi.2003, J. Ambrose (1, DEBU), 00137345. 
Tribe Scolytini Latreille, 1804

Scolytus multistriatus (Marsham, 1802), new data on first Alberta record

Note. This record of this adventive Palaearctic species was reported by Bright and Skidmore (1997) without specimen data. Scolytus multistriatus is a pest of Ulmus spp., and also known from British Columbia, Manitoba, Ontario, Nova Scotia, Quebec, and Saskatchewan.

Specimen data. Alberta: Calgary, $51.050^{\circ} \mathrm{N}, 114.084^{\circ} \mathrm{W}, 19 . v i i .1994, \mathrm{~T}$. Reichardt (2, CNCI), CNC Diptera 134316 to 134317; Edmonton, $53.554^{\circ} \mathrm{N}$, 113.406º W, 6.vii.-14.viii.1995, C. Brososky (1, CNCI), CNC Diptera 134314.

\section{Scolytus oregoni Blackman, 1934, new to Canada}

Note. This infrequently collected native species breeds in Pseudotsuga sp. in USA (Wood 1982).

Specimen data. British Columbia: Vancouver Island, Victoria, $48.541^{\circ} \mathrm{N}$, 123.469 $\mathrm{W}, 11-25 . v i i i .2009$, ex. funnel trap, CFIA, (3, CNCI).

\section{Scolytus piceae (Swaine, 1910), new to Newfoundland}

Note. This infrequently collected native species breeds mainly in Picea spp. (Bright 1976). It is otherwise known from all other provinces and territories except for Prince Edward Island.

Specimen data. Newfoundland: Humber District-Corner Brook, 12 mi NE Deer Lake, $49.318^{\circ} \mathrm{N}, 57.212^{\circ} \mathrm{W}$, 23.vii.1970, ex. Picea mariana, D.E. Bright (1, CNCI), CNC Diptera 133720.

\section{Scolytus rugulosus (Müller, 1818), new data on first Canadian record}

Note. This adventive Palaearctic species was apparently reported from Canada in Ontario by Chamberlin (1939) without specimen data. It breeds in Malus, Prunus and Pyrus trees.

Specimen data. Ontario: Prince Edward Co., 2.vii.1917, J.F. Brimley (1, CNCI), CNC Diptera 133735.

Scolytus schevyrewi Semenov Tjan-Shansky, 1902, new to Alberta, Saskatchewan, Manitoba, and Ontario

Note. This adventive Palaearctic species is known from British Columbia (Humble et al. 2010), and breeds in elms, particularly damaging the adventive Ulmus pumila (Ulmaceae). 
Specimen data. Alberta: Medicine Hat, Gershaw Avenue, 1-30.ix.2006, ex. funnel trap, CFIA (1, CNCI), CNC COLEO 00105817; Manitoba: RM De Salaberry Otterburne, $49.973^{\circ} \mathrm{N}, 97.052^{\circ} \mathrm{W}, 2007$, ex. funnel trap, CFIA, (2, CNCI), CNC COLEO 00106339, 00106340; Ontario: RM Peel, Mississauga, $43.711^{\circ} \mathrm{N}, 79.722^{\circ} \mathrm{W}, 6-19 . v i i i .2008$, ex. funnel trap, CFIA (1 CNCI); RM Hamilton, $43.269^{\circ} \mathrm{N}, 79.83^{\circ} \mathrm{W}, 29 . v .-11 . v i .2012$, ex. funnel trap, CFIA (1, no voucher retained); Lambton Co., Sarnia, $43.986^{\circ} \mathrm{N}, 82.410^{\circ} \mathrm{W}, 11-25 . v i ., 30 . v i .-11$. vii.2012, ex. funnel trap, CFIA (2, CNCI); Saskatchewan: Maple Creek, $49.917^{\circ} \mathrm{N}$, 109.484 ${ }^{\circ} \mathrm{W}, 21 . i v .2007$, ex. funnel trap, CFIA (1, CNCI), CNC COLEO 00105818; Regina, v.-vii.2007, ex. funnel trap, CFIA (1, CNCI), CNC COLEO 00106339 (specimens also examined from Assiniboia, Eston, Estevan, Moose Jaw, Shaunavon, Weyburn, Yorkton).

\section{Tribe Xyleborini LeConte, 1876}

Members of this tribe are obligate symbionts of fungi, which they introduce and cultivate in the xylem of their woody hosts.

\section{Euwallacea validus (Eichhoff, 1875), new to Canada}

Note. This adventive species has been present in North America since 1976 and has since spread within the eastern USA (Rabaglia et al. 2006). Euwallacea validus breeds in a variety of broadleaved and conifer trees, and its pest-status remains unclear.

Specimen data. Ontario: Essex Co., Windsor, Bloomfield \& Watkins, $42.291^{\circ} \mathrm{N}$, $83.076^{\circ} \mathrm{W}, 4 . i x .2004$, ex. Ailanthus altissima, E. Czerwinski (2, CNCI), CNC COLEO 00105866, 00105867; Niagara, Douglastown, $42.974^{\circ} \mathrm{N}, 79.018^{\circ} \mathrm{W}, 22 . x i .2005$, ex. Pinus sylvestris, L. Tucker (1, CNCI), CNC COLEO 00106198.

\section{Xyleborinus attenuatus (Blandford, 1894), new to Nova Scotia, Ontario and Quebec}

Note. This adventive species, which was until recently known mainly by the synonym $X$. alni Niisima, is a recent arrival in North America (Mudge et al. 2001). It was found soon after initial detection in much of NE and NW USA and British Columbia and Prince Edward Island. Xyleborinus attenuatus feeds on woody angiosperms, and its pest-status remains unclear.

Specimen data. Nova Scotia: Halifax, $44.738^{\circ} \mathrm{N}, 63.546^{\circ} \mathrm{W}$, 29.v.-12.vi.2007, ex. funnel trap, CFIA (2, CNCI), CNC COLEO 00106125, 00106126; Ontario: Middlesex Co., London, $42.983^{\circ} \mathrm{N}, 81.233^{\circ} \mathrm{W}, 27 . i v .1998$, ex. funnel trap, CFIA (2, CNCI), CNC COLEO 00106144, 00106145; Quebec: Sherbrooke, $45.417^{\circ} \mathrm{N}, 71.900^{\circ} \mathrm{W}$, 20.v.-2.vi.2009, ex. funnel trap, CFIA (1, CNCI), CNC COLEO 00106128. 


\section{Xyleborus affinis Eichhoff, 1868, new to Quebec}

Note. This apparently native species is already known from neighbouring Ontario and

New York State (Rabaglia et al. 2006), and breeds in deciduous trees.

Specimen data. Quebec: MRC Deux-Montagnes, Oka, Parc d'Oka, Lac de la Sauvagine, 2.xi.2002, R. Vigneault (2 CNCI; 23, CRVI), CNC Diptera 125448, 125449.

\section{Xyleborus celsus Eichhoff, 1868, new to Canada}

Note. This native species breeds in Carya spp. (Juglandaceae) in the USA (Rabaglia et al. 2006). In Canda these trees occur in only southern parts of Ontario and Quebec.

Specimen data. Ontario: Kent Co., Rondeau Provincial Park, $42.329^{\circ} \mathrm{N}$, $81.846^{\circ} \mathrm{W}$, 9.vi.1980, H. Goulet (1, CNCI), CNC Diptera 125451; Kent Co., Rondeau Provincial Park, Visitor's Centre, $42.781^{\circ} \mathrm{N}, 81.844^{\circ} \mathrm{W}, 3 . v i i .2003$, S.M. Paiero (1, DEBU), debu01119222; Middlesex Co., London, $43.090^{\circ} \mathrm{N}, 81.187^{\circ} \mathrm{W}$, 13.viii.2006, ex. sticky trap, K. Nystrom (1, CNCI; 1, GLFC), CNC COLEO 00106117.

\section{Xyleborus ferrugineus (Fabricius, 1801), new to Canada}

Note. This apparently native species, feeds in a wide variety of woody plants, and is known in the USA from nearby Michigan, New York, Ohio and Pennsylvania, and is also present as an adventive on other continents (Rabaglia et al. 2006).

Specimen data. Ontario: Kent Co., Rondeau Provincial Park, 11-25.v.1985, flight intercept trap in maple-beech forest, L. Lesage and A. Woodliffe, (1, CNCI).

\section{Xylosandrus crassiusculus (Motschulsky 1866), new to Canada}

Note. This east-Asian species is known from throughout the eastern USA (Rabaglia et al. 2006), including nearby Ohio (Lightle et al. 2007) and Michigan (Cognato et al. 2009), from as far north as $45.350^{\circ} \mathrm{N}$. This species breeds in many broadleaved woody plants and is a pest in the USA in apparently healthy nursery material and fruit trees. (Kovach and Gorsuch 1985, Oliver and Mannion 2001)

Specimen data. Ontario: Elgin Co., $42.826^{\circ} \mathrm{N}, 81.288^{\circ} \mathrm{W}, 11-25 . v i ., 12-26 . v i i .$, 10-24.viii., 18.ix.-2.x.2012, ex. funnel trap, CFIA (27, CNCI).

\section{Xylosandrus germanus (Blandford, 1894), new data on first Quebec record}

Note. This adventive Palearctic species was first reported from Quebec by Bright and Skidmore (2002) without reference to specimens. The following is to document these 
first-known captures of this species in Quebec. Xylosandrus germanus feeds in both broad leaved and conifer trees.

Specimen data. Quebec: MRC Longueuil, Saint-Bruno-de-Montarville, Mont St-Bruno, $45.55^{\circ} \mathrm{N}, 73.316^{\circ} \mathrm{W}, 30 . v .-5 . v i ., 5-12 . v i ., 12-19 . v i .$, 19-26.vi., 26.vi.-2. vii., 2-12.vii., 12-17.vii., 17-24.vii., 24.vii.-1.viii., 1-8.viii.2000, G. Pelletier (18 CNCI), CNC Diptera 128373 to 128391.

\section{Discussion}

McNamara (1991) provided a comprehensive list of Curculionoidea known from Canada and its provinces at that time. Noteworthy additions to our knowledge of the Canadian weevil fauna in the last 20 years include the works published by Bright $(1993,1994)$, Bouchard et al. (2005), Bright and Skidmore (1997), Anderson (1997), Chantal (1998), Anderson (2002), Anderson and Korotyaev (2004), Rabaglia et al. (2006), Majka and Anderson (2007), Majka et al. (2007a-c), Bright and Bouchard (2008), Humble et al. (2010), Klimaszewski et al. (2010), De Clerck-Floate and Cárcamo (2011), Webster et al. (2012), Bouchard et al. (2012), Humble and Hueppelsheuser (2012) and Looney et al. (2012). Beyond these, we list 24 species new to Canada, in ten cases also representing new records at the generic level. We have also added 59 species new to 12 provinces and territories. These records include 10 pest species and six species introduced elsewhere as biological control agents. Some are new records of adventive species expanding their range, others may be northward expansions of species common in the USA, and many fill gaps within the patchy known distributions of infrequently collected native species.

The present review of collections material for new distributional records was undertaken in anticipation of the new checklist of Canadian Coleoptera (Bousquet et. al 2013). Except for the maratimes provinces, which are now receiving increased faunistic research (e.g. Majka and Anderson (2007), Majka et al. (2007a-c), Webster et al. (2012)), our understanding of most other beetle families would also benefit from such a review. It is also probable that further undocumented curculionoid first records remain to be gleaned from material at Canadian insect collections which we were unable to include in this study.

The range extension for Asperosoma echinatum from Manitoba into southern Ontario is partidularly interesting. It is very uncommon for species to be endemic to Canada but this is an example where both the genus and species are, at present, known only from Canada. Possible other host plants in the genus Heuchera (Saxifragiaceae) are widespread in North America and the weevil may be more widely distributed than currently known. Whether or not a Canadian endemic, it is a little-known and possibly at-risk species that is worth searching for.

\section{Acknowledgements}

This work represents the research activities of many people and organizations: field collecting, sorting trap samples, preliminary identifications, and providing loans, as 
part of their ongoing efforts to document their regional insect fauna. Some bark beethe records are based on CNCI specimens that D.E. Bright had identified. The authors thank I. Nei and K. McLachlan-Hamilton for assisting with identifications; Jeff Boone (City of Saskatoon), Stéphanie Boucher (LEMQ), Claude Chantal (CCCH), CFIA's Plant Health Survey Unit and inspectors, Tom Hutchison and Sunil Ranasinghe (Alberta Environment and Sustainable Resource Development), Serge Laplante (CSLA), Rory McIntosh (Saskatchewan Ministry of Environment), Kathryn Nystrom (Great Lakes Forestry Centre), Steven Paiero (DEBU), and Irene Pines (Manitoba Conservation) for providing specimens (often already identified). We thank Chantal Alderson, Vincent Webster and Cory Hughes for assistance in collecting Coleoptera in New Brunswick. Jon Sweeney (Natural Resources Canada, Canadian Forest Service), CFIA, and USDA APHIS are thanked for funding surveys in New Brunswick. Bruce Gill,Troy Kimoto, D. McQuorquodale, and one anonymous reviewer provided helpful comments on the manuscript.

\section{References}

Ahmad M, Burke HR (1972) Larvae of the weevil tribe Anthonomini (Coleoptera: Curculionidae). Miscellaneous Publications of the Entomological Society of America, 8: 33-81.

Alonso-Zarazaga MA (2011) Family Apionidae Schoenherr, 1823. In: Löbl I, Smetana A. (Eds) Catalogue of Palaearctic Coleoptera. Volume 7. Curculionoidea I. Apollo Books, Stenstrup, 148-176.

Anderson RS (1997) Weevils (Coleoptera: Curculionoidae, excluding Scolytinae and Platypodinae) of the Yukon, 523-562 pp. In: Danks HV and Downes JA (Eds) Insects of the Yukon. Biological survey of Canada (Terrestrial Arthropods), Ottawa, 1034 pp.

Anderson RS (2002) Chapter 131. Curculionidae. In: Arnett RH Jr, Thomas MC, Skelley P (Eds) American Beetles Volume 2. CRC Press, Gainesville Florida, 722-815.

Anderson RS (2003) Pseudotychius watsoni Blatchley (Curculionoidea; Nanophyidae) new to Canada. The Coleopterists Bulletin 57: 110-122. doi: 10.1649/0010-065X(2003)057[0 110:SN]2.0.CO;2

Anderson RS (2008) A review of the genus Cryptorhynchus Illiger 1807 of North America (Coleoptera: Curculionidae; Cryptorhynchinae). The Coleopterists Bulletin, 62: 168-180. doi: $10.1649 / 1065.1$

Anderson RS, Korotyaev BA (2004) Some Palearctic weevils in the subfamily Ceutorhynchinae (Coleoptera, Curculionidae) recently discovered in North America. The Canadian Entomologist 136: 233-239. doi: 10.4039/n03-064

Anderson RS, O’Brien CW, Salsbury GA, Krauth SJ (2007) Orchestes alni (L.) newly discovered in North America (Coleoptera: Curculionidae). Journal of the Kansas Entomological Society 80: 78-79. doi: 10.2317/0022-8567(2007)80[78:OALNDI]2.0.CO;2

Blatchley WS, Leng CW (1916) Rhynchophora or Weevils of North Eastern America. The Nature Publishing Company, Indianapolis, 682 pp. doi: 10.5962/bhl.title.1557 
Bouchard P, Bousquet Y, Davies AE, Alonso-Zarazaga MA, Lawrence JF, Lyal CHC, Newton AF, Reid CAM, Schmitt M, Ślipiński SA, Smith ABT (2011) Family-group names in Coleoptera (Insecta). ZooKeys 88: 1-972. doi: 10.3897/zookeys.88.807

Bouchard P, Gagnier DL, Hunt DWA (2012) Cercopeus schwarzi Sleeper, 1955 (Coleoptera: Curculionidae: Entiminae) new to Canada. The Coleopterists Bulletin 66: 116-117. doi: $10.1649 / 072.066 .0204$

Bouchard P, LeSage L, Goulet H, Bostanian NJ, Vincent C, Zmudzinska A, Lasnier J (2005) Weevil (Coleoptera: Curculionoidea) diversity and abundance in two Quebec vineyards. Annals of the Entomological Society of America 98: 565-574. doi: 10.1603/0013-8746(2005)098[0565:WCCDAA]2.0.CO;2

Bousquet Y, Bouchard P, Davies AE, Sikes DS (2013) Checklist Of Beetles (Coleoptera) Of Canada And Alaska. Second Edition. Data Paper. Zookeys X: X-XX.

Bright DE (1976) The Bark Beetles of Canada and Alaska. Coleoptera: Scolytidae. The insects and arachnids of Canada. Part 2. Agriculture Canada, Ottawa 241 pp.

Bright DE (1993) The insects and arachnids of Canada. Part 21. The weevils of Canada and Alaska. 1. Coleoptera: Curculionidea, excluding Scolytidae and Curculionidae. Agriculture Canada Publication 1882: 217 pp.

Bright DE (1994) Revision of the genus Sitona (Coleoptera: Curculionidae) of North America. Annals of the Entomological Society of America 87: 277-306.

Bright DE, Bouchard P (2008) Weevils of Canada and Alaska. Volume 2. Coleoptera, Curculionidae, Entiminae. NRC Research Press, Ottawa, i-xiii + 327 pp.

Bright DE, Skidmore RE (1997) A catalog of Scolytidae and Platypodidae (Coleoptera). Supplement 1 (1990-1994) National Research Council Research Press, Ottawa, 368 pp.

Bright DE, Skidmore RE (2002) A Catalog of Scolytidae and Platypodidae (Coleoptera): Supplement 2 (1995-1999) National Research Council Research Press, Ottawa, 523 pp.

Buchanan LL (1946) Notes on American Rhyncolus, with description of a new species (Coleoptera, Curculionidae). Bulletin of the Brooklyn Entomological Society 41: 129-136.

Chamberlin WJ (1939) The bark and timber beetles of North America north of Mexico. The taxonomy, biology and control of 575 species belonging to 72 genera of the superfamily Scolytoidea. Oregon State Cooperative Association, Corvallis, Oregon, 513 pp.

Chantal C (1992) Première mention Canadienne de Micracis suturalis LeConte (Coleoptera: Scolytidae). Fabreries 17: 61-62.

Chantal C (1998) Quatre premières mentions de charançons (Coléoptèra: Curculionidae) pour le Québec. Fabreries 23: 105.

Ciegler JC (2010) Weevils of South Carolina (Coleoptera: Nemonychidae, Attelabidae, Brentidae, Ithyceridae, and Curculionidae). South Carolina Agriculture and Forestry Research System, Clemson Public Service Activities, 276 pp.

Clark WE (1980) Revision of Nearctic weevils of the genus Lignyodes Dejean (Coleoptera: Curculionidae). Transactions of the American Entomological Society 106: 273-326.

Clark WE (1987) Revision of the Nearctic species of Pseudanthonomus Dietz (Coleoptera: Curculionidae). The Coleopterists Bulletin 41: 263-285.

Cognato AI, Barc N, Philip M, Mech R, Smith AD, Galbraith E, Storer AJ, Kirkendall LR (2009) The Native and Introduced Bark and Ambrosia Beetles of Michigan (Coleoptera: Curculionidae, Scolytinae). The Great Lakes Entomologist 42: 101-120. 
De Clerck-Floate RA, Cárcamo HA (2011) Chapter 12. Biocontrol arthropods: new denizens of Canada's grassland agroecosystems. In: Floate KD (Ed) Arthropods of Canadian Grasslands. Vol. 2: Inhabitants of a Changing Landscape, Biological Survey of Canada, 291-321.

Fall HC (1917) New Coleoptera - VII. The Canadian Entomologist 49: 385-391. doi: 10.4039/Ent49385-11

Gibson LP (1969) Monograph of the genus Curculio in the New World (Coleoptera: Curculionidae). Part I. United States and Canada. Miscellaneous Publications of the Entomological Society of America 6: 239-285.

Humble LM, John E, Smith J, Zilahi-Balogh G, Kimoto T, Noseworthy MK (2010) First records of the banded elm bark beetle Scolytus schevyrewi (Semenov) (Coleoptera: Curculionidae: Scolytinae), in British Columbia. Journal of the Entomological Society of British Columbia 107: 21-24.

Humble LM, Hueppelsheuser T (2012) First record of Polydrusus sericeus (Schaller) (Coleoptera: Curculionidae) in western North America. The Coleopterists Bulletin 66: 72-73.

Klimaszewski J, Langor D, Majka CG, Bouchard P, Bousquet Y, LeSage L, Smetana A, Sylvestre P, Pelletier G, Davies A, DesRochers P, Goulet H, Webster R, Sweeney J (2010) Review of adventive species of Coleoptera (Insecta) recorded from eastern Canada. Pensoft, Bulgaria. 272 pp.

Kovach J, Gorsuch CS (1985) Survey of ambrosia beetle species infesting South Carolina peach orchards and a taxonomic key for the most common species. Journal of Agricultural Entomology 2: 238-247.

Lightle D, Gandhi K, Cognato A, Mosley B, Nielsen D, Herms D (2007) New reports of exotic and native ambrosia and bark beetle species (Coleoptera: Curculionidae: Scolytinae) from Ohio. The Great Lakes Entomologist 40: 194-200.

Looney C, Humble LM, Cranshaw W (2012) Orchestes alni (L.) (Coleoptera: Curculionidae): new records from western North America with notes on parasitoids. The Coleopterists Bulletin 66: 63-66. doi: 10.1649/072.066.0115

Majka CG, Anderson RS (2007) The genus Tropiphorus (Coeloptera: Curculionidae) in North America. The Coleopterists Bulletin 61: 487-489. doi: 10.1649/0010-065X(2007)61[48 7:TGTCCE] 2.0.CO;2

Majka CG, Anderson RS, McAlpine DF, Webster RP (2007a) The weevils (Coleoptera: Curculionoidea) of the Maritime Provinces of Canada, I: New records from New Brunswick. The Canadian Entomologist 139: 378-396. doi: 10.4039/n06-008

Majka CG, Anderson RS, McCorquodale DB (2007b) The weevils (Coleoptera: Curculionoidea) of the Maritime Provinces of Canada, II: New records from Nova Scotia and Prince Edward Island and regional zoogeography. The Canadian Entomologist 139: 397442. doi: 10.4039/n06-021

Majka CG, Anderson RS, Georgeson E (2007c) Introduced Apionidae and Brentidae (Coleoptera: Curculionoidea) in the Maritime Provinces of Canada. Proceedings of the Entomological Society of Washington 109: 66-74.

McNamara J (1991) Superfamily Curculionoidea. In Bousquet Y (ed) Checklist of the beetles of Canada and Alaska. Agriculture Canada, Ottawa, 323-365.

Mudge AD, LaBonte JR, Johnson KJR, LaGasa EH (2001) Exotic woodboring Coleoptera (Micromalthidae, Scolytidae) and Hymenoptera (Xiphydriidae) new to Oregon and Washington. Proceedings of the Entomological Society of Washington 103: 1011-1019. 
O'Brien CW, Wibmer G (1982) Annotated checklist of the weevils (Curculionidae sensu lato) of North America, Central America, and the West Indies. Memoirs of the American Enfornological Institute 34: IX. 382 pp.

Oliver JB, Mannion CM (2001) Ambrosia beetle (Coleoptera: Scolytidae) species attacking Chestnut and captured in ethanol-baited traps in middle Tennessee. Environmental Entomology 30: 909-918. doi: 10.1603/0046-225X-30.5.909

Piper GL (1977) Biology and immature stages of Cylindrocopturus quercus (Say) (Coleoptera: Curculionidae). The Coleopterists Bulletin 31: 65-72.

Rabaglia RJ, Dole SA, Cognato AI (2006) Review of American Xyleborina (Coleoptera: Curculionidae: Scolytinae) occurring North of Mexico, with an illustrated key. Annals of the Entomological Society of America 99: 1034-1056. doi: 10.1603/0013-8746(2006)99[1034:RO AXCC]2.0.CO;2

Turkington R, Franko GD (1980) The biology of Canadian weeds. 41. Lotus corniculatus L. Canadian Journal of Plant Science 60: 965-979. doi: 10.4141/cjps80-140

Turkington R, Cavers PB, Rempel E (1978) The biology of Canadian weeds. 29. Melilotus alba Desr. and M. officinalis (L.) Lam. Canadian Journal of Plant Science 58: 523-537. doi: $10.4141 /$ cjps78-078

Valentine BD (1999) A review of Nearctic and some related Anthribidae (Coleoptera). Insecta Mundi 12: 251-296.

Vaurie P (1951) Revision of the genus Calendra (formerly Sphenophorus) in the United States and Mexico (Coleoptera, Curculionidae). Bulletin of the American Museum of Natural History 98: 33-186.

Webster RP, Anderson RS, Sweeney JD, DeMerchant I (2012) New Coleoptera records from New Brunswick, Canada: Anthribidae, Brentidae, Dryophthoridae, Brachyceridae, and Curculionidae, with additions to the fauna of Quebec, Nova Scotia and Prince Edward Island. ZooKeys 179: 349-406. doi: 10.3897/zookeys.179.2626

Wheeler AG Jr, Hoebeke ER (2009) Chapter 21. Adventive (Non-native) Insects: Importance to Science and Society. In: Foottit RG. Adler PH (Eds.) Insect Biodiversity: Science and Society. Blackwell Publishing, New Jersey, 475-522.

Wibmer GJ (1981) Revision of the New World weevil genus Tyloderma in America north of Mexico (Coleoptera: Curculionidae: Cryptorhynchinae). Southwestern Entomologist Supplement 3: 1-95.

Wood SL (1982) The bark and ambrosia beetles of North and Central America (Coleoptera: Scotytidae), a taxonomic monograph. The Great Basin Naturalist Memoirs No 6. 1359 pp. Wood SL, Bright DE (1992) A catalog of Scolytidae and Platypodidae (Coleoptera), Part 2: Taxonomic Index, Great Basin Naturalist Memoirs No. 13. Brigham Young University, Provo, UT, USA. 1553 pp. 